Check for updates

Cite this: Mater. Chem. Front. 2020, 4, 449

Received 29th October 2019 Accepted 12th December 2019

DOI: $10.1039 / c 9 q m 00667 b$

rsc.li/frontiers-materials

\title{
Recent advances and prospects of carbon dots in cancer nanotheranostics
}

\author{
Qingyan Jia, ${ }^{\text {abc }}$ Ziyu Zhao, ${ }^{\text {bc }}$ Ke Liang, ${ }^{\text {bc }}$ Fuchun Nan, ${ }^{\text {bc }}$ Ye Li, ${ }^{\text {bc }}$ Jian Wang, ${ }^{\text {bc }}$ \\ Jiechao Ge (D)*bc and Pengfei Wang*bc
}

\begin{abstract}
As a new member of the carbonaceous nanomaterial family, carbon dots (CDs) have been recognized as promising candidates in nanotheranostics for simultaneous bioimaging and cancer treatment because of their unique optical properties and intrinsic theranostic properties. In this short review, we aim at summarizing the recent advances and future prospects of CDs as nanotheranostic agents for anticancer applications. Initially, we discuss CDs for fluorescence imaging, photoacoustic imaging and magnetic resonance imaging. Subsequently, we highlight CDs for photodynamic therapy, photothermal therapy, multimodal therapy, and tumor microenvironment stimulus-responsive cancer treatment. Lastly, we discuss the present challenges and future prospects of CDs as versatile nanotheranostic agents for cancer treatment.
\end{abstract}

\section{Introduction}

Cancer has become a primary health burden in the world due to its high incidence and mortality. Despite remarkable efforts in cancer treatment, the overall 5 year survival rate of patients with cancer is still very low. ${ }^{1}$ Around 18 million new cancer cases and approximately 9.6 million deaths from cancer reported in 2018 ,

${ }^{a} X i$ 'an Institute of Flexible Electronics (IFE) \& Xi'an Institute of Biomedical Materials and Engineering (IBME), Northwestern Polytechnical University (NPU), 127 West Youyi Road, Xi'an 710072, China

${ }^{b}$ Key Laboratory of Photochemical Conversion and Optoelectronic Materials and CityU-CAS Joint Laboratory of Functional Materials and Devices, Technical Institute of Physics and Chemistry, Chinese Academy of Sciences, Beijing, 100190, China.E-mail: jchge2010@mail.ipc.ac.cn,wangpf@mail.ipc.ac.cn

'School of Future Technology, University of Chinese Academy of Sciences, Beijing 100049, China

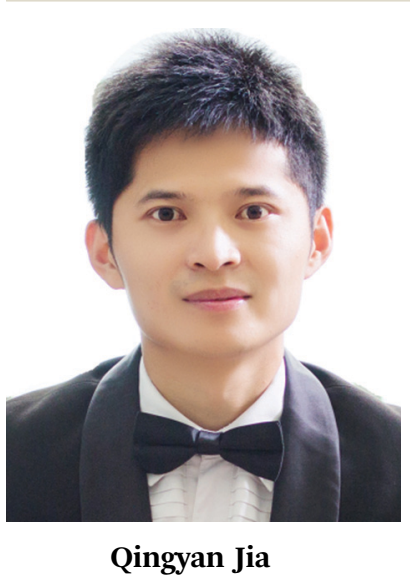

Qingyan Jia is an associate professor at Northwestern Polytechnical University in China. He received his $P h D$ degree from Technical Institute of Physics and Chemistry (TIPC) in 2018. In 2018, he joined the Institute of Flexible Electronics (IFE) at Northwestern Polytechnical University. The primary goal of his research team is to develop novel nanomaterials and innovative strategies for cancer and infection treatments. and the annual number of cancer cases will have increased to 22 million in the next two decades. Therefore, new medicines and techniques must be developed for precise and efficient cancer therapy.

Nanotheranostics that integrate diagnosis and therapy into a single nanoplatform have attracted increasing interest for cancer treatment. ${ }^{2-5}$ In comparison with traditional anticancer models, the combination of diagnosis and therapy can collect more detailed information for precise, high-sensitivity, and low-interference cancer treatment. The integration also can monitor the metastasis and relapse of tumor to obtain high therapeutic efficacy. Hence, a large variety of nanotheranostic agents based on multicomponent inorganic, organic, or inorganic/ organic nanostructures that combine two or more imaging functions (e.g., fluorescence (FL)/photoacoustic (PA) imaging, $\mathrm{FL} /$ magnetic resonance (MR) imaging, MR/positron emission computed tomography (PET) imaging, or FL/MR/PET imaging) and therapeutic modalities (e.g., chemotherapy (CHT)/radiotherapy (RT), gene therapy (GT)/photodynamic therapy (PDT), CHT/photothermal therapy (PTT), and PDT/PTT/CHT) have been studied in quantities, and many interesting results have been obtained in preclinical animal experiments on various tumor models. ${ }^{6-10}$ Although these techniques have gained prominence, each type of nanotheranostic agents has unique properties and intrinsic limitations. Inorganic nanoparticles, such as transition metal chalcogenide or selenide nanoparticles and upconversion nanoparticles, often have unique physicochemical properties that allow applications in simultaneous imaging and therapy of cancer. Unfortunately, the use of these particles in clinical settings is impeded by the long-term safety of heavy metal atoms associated with these nanoparticles. Research on nanotheranostics 
derived from low toxicity materials is still in infancy, and using fewer components to develop new and safe/biocompatible "one for all" theranostic features for clinical multimodal imaging and therapeutic modalities remains a major challenge.

In the past decade, carbon dots (CDs) have attracted considerable attention in different fields, including bioimaging, photocatalysis, light-emitting diodes, and plant growth promotion, because of their remarkable nanostructures and favorable electronic, mechanical, optical, thermal, and safe/biocompatible properties. ${ }^{11-17}$ CDs were first discovered accidentally in 2004 by $\mathrm{Xu}$ and coworkers, and then Dr Sun named them CDs or carbon quantum dots (CQDs) based on their spectral features and properties that are similar to those of the widely studied silicon QDs at that time. ${ }^{18,19}$ The preparation methods of CDs are currently divided into bottom-up and top-down approaches. The bottom-up approach uses a variety of technologies, such as template method, pyrolysis, microwave, solvothermal, etc. to carbonize organic molecules or biomass containing organic molecules to obtain emitting CDs. The top-down approach is to prepare smaller luminescent CDs with large bulk carbon

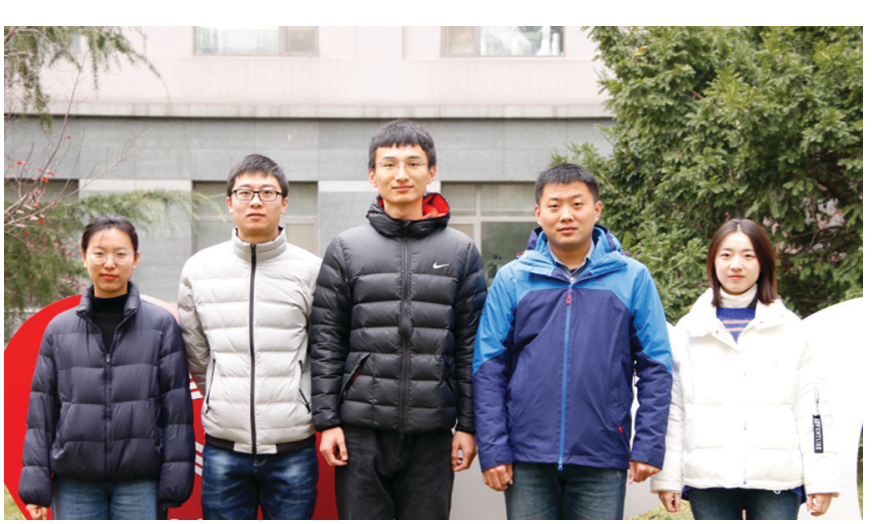

From left to right: Ye Li, Jian Wang, Ke Liang, Fuchun Nan, and Ziyu Zhao materials, such as graphite, carbon fiber, carbon nanotubes, graphene, etc. as precursors by using laser ablation, electrochemistry, arc discharge, and other technologies. ${ }^{20,21}$ According to the reported literature, CDs mainly include CQDs, graphene quantum dots (GQDs), polymer carbon nanodots, and carbon nanodots. $^{22-25}$ CDs can effectively overcome the metal toxicity of traditional II-VI QDs; thus, they can be applied as highly stable/ biocompatible fluorescence probes and biological tracers in vivo. Moreover, fluorescent CDs are superior to organic dyes in terms of photoluminescence stability, fluorescence tunability, surface group richness, strong hydrophilicity, low toxicity, good biocompatibility, rich carbon source, and simple preparation. Therefore, CDs have been recognized as smart candidates for the diagnosis of cancer.

In addition to the use of FL imaging, a variety of CDs have been studied for PA imaging, MR imaging, etc for cancer diagnosis. Some CDs also have inherent anticancer properties, including PDT and PTT. ${ }^{26-29}$ PDT is mainly to use photosensitizers (PSs) to produce reactive oxygen species (ROSs) under proper light illumination, and then generate oxidation

Ziyu Zhao, Ke Liang, Fuchun Nan, Ye Li, and Jian Wang are students at Key Laboratory of Photochemical Conversion and Optoelectronic Materials and CityU-CAS Joint Laboratory of Functional Materials and Devices of Technical Institute of Physics and Chemistry of Chinese Academy of Sciences. Ye Li and Ziyu Zhao are studying for their Master's degree. Ke Liang, Fuchun Nan, and Jian Wang are pursing for their PhD degree. They are focusing on the development of carbon nanomaterials for biomedical, energy, and environmental applications.

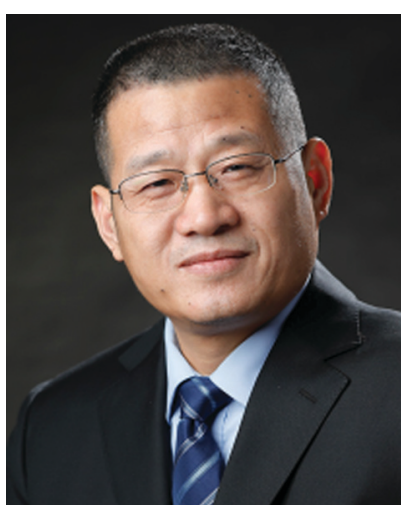

Jiechao Ge
Jiechao Ge is currently a professor at the TIPC, Chinese Academy of Sciences. He received his $P h D$ degree from Shandong Normal University in 2008 under the guidance of Prof. Bo Tang. During 2008 and 2009, he joined the group of Prof. Harry Dorn and worked as a postdoctoral researcher at Virginia Polytechnic Institute \& State University. He joined TIPC in 2010. His research interests mainly focus on the design and synthesis of carbon nanomaterials as cancer theranostics and photocatalysts.

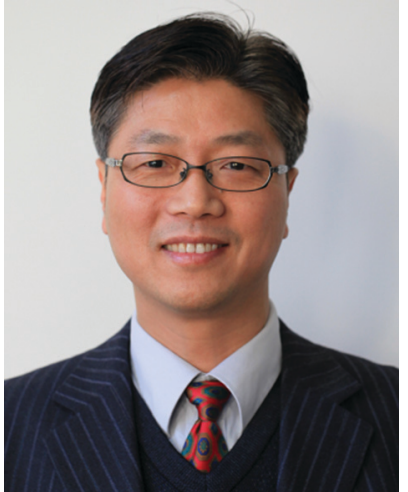

Pengfei Wang
Pengfei Wang is a professor at TIPC. $\mathrm{He}$ received his $\mathrm{PhD}$ degree in organic chemistry from the Institute of Photographic Chemistry, CAS, in 1993. After completing professional experience at Laboratoire PPSM of CNRS of France as a visiting researcher, at the National Institute of Materials and Chemical Research (NIMC) in Japan as a COE fellow, and at the Department of Applied Physics and Materials Science in City University of Hong Kong as a senior research fellow, he joined

TIPC in 2005 as a professor. His current research interests include chemosensors and phototheranostic agents based on organic/inorganic materials. 
reaction with biological macromolecules in cancer cells, resulting in cell toxicity; PTT is usually to absorb photon energy through photothermal agents, and then release heat in the tumor site inducing irreversible damage to cancer cells. The light illumination in PDT and PTT are able to be spatially controlled to irradiate only the tumor lesion without damaging normal tissues. Such selectivity offered by PDT and PTT can significantly reduces the systemic toxicity compared with the traditional chemotherapy and radiotherapy. ${ }^{30,31}$ Thus, the CDs with intrinsic phototheranostic properties have shown considerable potential for application in tumor treatment. Although several articles have reviewed CD syntheses and applications, including imaging, diagnosis, and cancer treatment, ${ }^{32-35}$ in the present review, we aim at summarizing the recent advances mainly reported in both 2018 and 2019 and the future prospects of CDs as nanotheranostic agents for anticancer applications. Initially, we discuss the present progress in the use of CDs for FL imaging, PA imaging as well as MR imaging. Subsequently, we highlight the recent advances in CD-based cancer nanotheranostics in branches of PDT, PTT, multimodal therapy, and tumor microenvironment stimulus-triggered therapy. Lastly, we present the current challenges and future prospects of CDs as versatile nanotheranostic agents for cancer treatment.

\section{Carbon dots for cancer diagnosis}

\subsection{Carbon dots as fluorescence imaging probes}

Fluorescent CDs have been widely used in bioimaging because of their excellent and tunable multicolor emission. For FL imaging probes, red or near-infrared (NIR) emissive CDs $(>600 \mathrm{~nm}$ ) are favorable due to their deep tissue penetration, minimal tissue absorption, minimal photodamage, and low autofluorescence interference to biological samples. ${ }^{34,36}$ However, prior to 2014, the reported CDs were mainly concentrated at a wavelength range of blue to yellow and generally excited using a short-wavelength light of less than $500 \mathrm{~nm}$, which is outside the biological optical window (650-900 nm); as a consequence, these CDs would be strongly absorbed by biological tissues, limiting light penetration in tissues. ${ }^{37,38}$ Therefore, the use of CDs as FL imaging probes for the in vivo diagnosis of cancer is hindered. In 2014, our group reported a novel $\mathrm{N}$ - and S-doped $\mathrm{CD}$ prepared from polythiophene derivative termed as PT2, which is a well-defined conjugated polymer molecular precursor. The CDs exhibit a broad absorption in the UV-visible region and a strong red emission peak at $680 \mathrm{~nm} .{ }^{39}$ Subsequently, we designed various functional precursors based on polythiophene derivatives to prepare CDs with tunable emissions ranging from blue to NIR (Fig. $1 \mathrm{a}$ and b). ${ }^{40}$ We also proposed a tunable photoluminescence mechanism resulting from variations in the surface state and $\mathrm{N}$ doping (Fig. 1c). The introduction of $\mathrm{N}$ atoms leads to a decrease of energy levels of the CDs' surface state. Moreover, the $S$ atom introduction likely enhances the effect of $\mathrm{N}$ atoms on the properties of CDs. These findings further prove that is an available way to improve the fluorescent properties and other

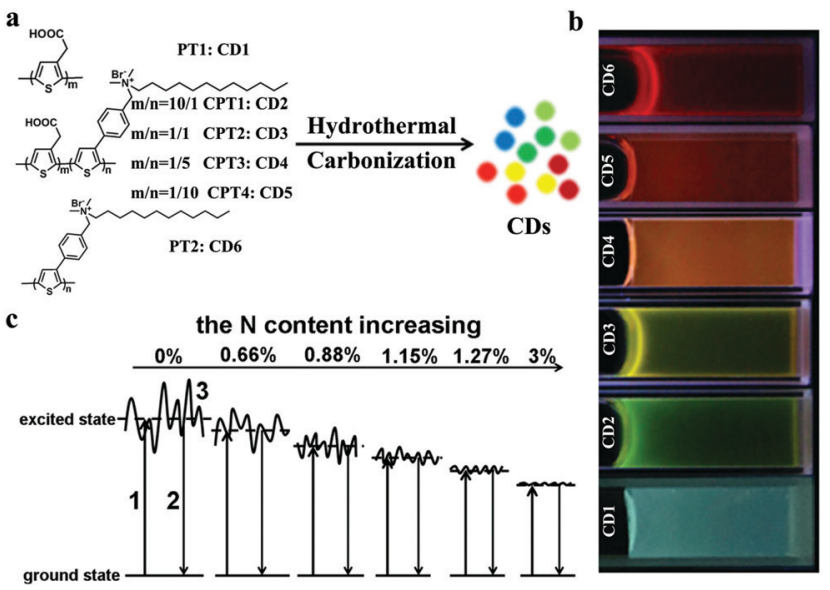

Fig. 1 (a) Synthetic routes of polythiophene derived CDs. (b) Emission colors of polythiophene-derived CDs under UV light. (c) FL mechanism of polythiophene-derived CDs: (1) electrons excited from the ground state; (2) excited electrons returning to the ground state; and (3) surface state of CDs. Reproduced with permission from ref. 40. Copyright 2016, the Royal Society of Chemistry.

physicochemical properties of CDs. ${ }^{41-43}$ Thus far, various heteroatoms, such as N, S, and F, with different doping methods have been utilized to explore CDs as bioimaging probes in the red/NIR emissive region (Table 1). For example, in 2016, Lin's group prepared highly efficient red emissive CDs by using citric acid and formamide as precursors. ${ }^{44}$ The red emissive CDs exhibit excellent properties, including high quantum yield (QY) of 22.9\% and red emission (maximum emission at $640 \mathrm{~nm}$ ). They found that formamide plays an important role in the favorable realization of red emissive CDs and concluded that the surface states/ defects of CDs and N/O codoping possibly cause the intense red emission. In 2017, Xiong et al. prepared red emissive CDs with a high QY of $28 \%$ in water by using pulp-free lemon juice. ${ }^{45}$ The red emissive CDs are monodispersed with an average diameter of $4.6 \mathrm{~nm}$ and exhibit excitation-independent emission with peak at $631 \mathrm{~nm}$. Further investigations have suggested that the surface states of CDs and the N-derived structures of CD cores synergistically cause the intense red emission. Subsequently, they prepared red emissive CDs with a high QY of $53 \%$ by heating citric acid and ethylenediamine in a formamide solution, thereby improving the wide applications of CDs in bioimaging. ${ }^{46}$ Fan's group also reported multicolored N-doped CDs by controlling the fusion and carbonization of an ethanol solution of citric acid and diaminonaphthalene (DAN) with a suitable amount of $\mathrm{H}_{2} \mathrm{SO}_{4} \cdot{ }^{47}$ Given the large $\mathrm{N}$-doped $\mathrm{sp}^{2}$ cluster of precursor DAN, the surface of CDs can be highly passivated and crystallized for yielding strong emission. Yang and coworkers fabricated one fluorine-doped CD with red photoluminescence via a simple and eco-friendly microwave-assisted method. ${ }^{48}$ The red-shift emission of CDs is mainly attributed to the doped fluorine, which increases the delocalized $\pi$-electron and then narrows the energy gap of the surface states of CDs. Additionally, Ding's group synthesized an NIR-emitted CD by using low-cost lemon juice as a precursor via a solvothermal reaction. ${ }^{49}$ The as-prepared CDs show excitation-independent emission with the 
Table 1 Heteroatom doped red emissive CDs prepared from different carbon sources and their emission properties

\begin{tabular}{|c|c|c|c|c|c|}
\hline Carbon source & Method & Doping & Emission $(\mathrm{nm})$ & QY (\%) & Ref. \\
\hline Citric acid, formamide & Microwave & $\mathrm{N}$ & 640 & 22.9 & 44 \\
\hline Pulp-free lemon juice & Solvothermal & $\mathrm{N}$ & 631 & 28 & 45 \\
\hline$N, N$-Dimethyl-, $N, N$-diethyl-, and $N, N$-dipropyl- $p$-phenylenediamine & Solvothermal & $\mathrm{N}$ & $637,642,645$ & 86 & 47 \\
\hline Citric acid, urea, sodium fluoride & Microwave & $\mathrm{N}, \mathrm{F}$ & 600 & 1.2 & 48 \\
\hline Pulp-free lemon juice, formamide & Solvothermal & $\mathrm{N}$ & 704 & 31 & 49 \\
\hline
\end{tabular}

highest fluorescent peak at $704 \mathrm{~nm}$ and a fluorescence QY of up to $31 \%$.

Surface functionalization is another effective strategy to improve the fluorescence of CDs. ${ }^{50-54}$ In 2018, Sun's group reported multiple-color-emission CDs from blue to red by controlling their surface graphitization and functionalization (Fig. 2a). ${ }^{55}$ The XRD (Fig. 2b) and Raman spectra (Fig. 2c) demonstrate that the graphitization degree of R-CDs is higher than those of B- and G-CDs. Fig. 2d shows that the $-\mathrm{COOH}$ content significantly increases from B- and G-CDs to R-CDs. Therefore, the emission peak of CDs can be tuned to $630 \mathrm{~nm}$ by regulating the thermal pyrolysis temperature and reactant ratio of citric acid and urea (Fig. 2e). The relative photoluminescence QYs of CDs with red emission can reach $12.9 \%$. Another notable progress was revealed by Qu and Rogach et al., who prepared NIR CDs by modifying polymers or molecules on their surface. ${ }^{56}$ For instance, they bound poly(vinylpyrrolidone) with electronacceptor groups, such as $\mathrm{S}-\mathrm{O}$ and $\mathrm{C}-\mathrm{O}$, to the outer layers and edges of CDs to facilitate the electron transitions between optical band gaps of CDs for NIR excitation and emission (Fig. 2f).
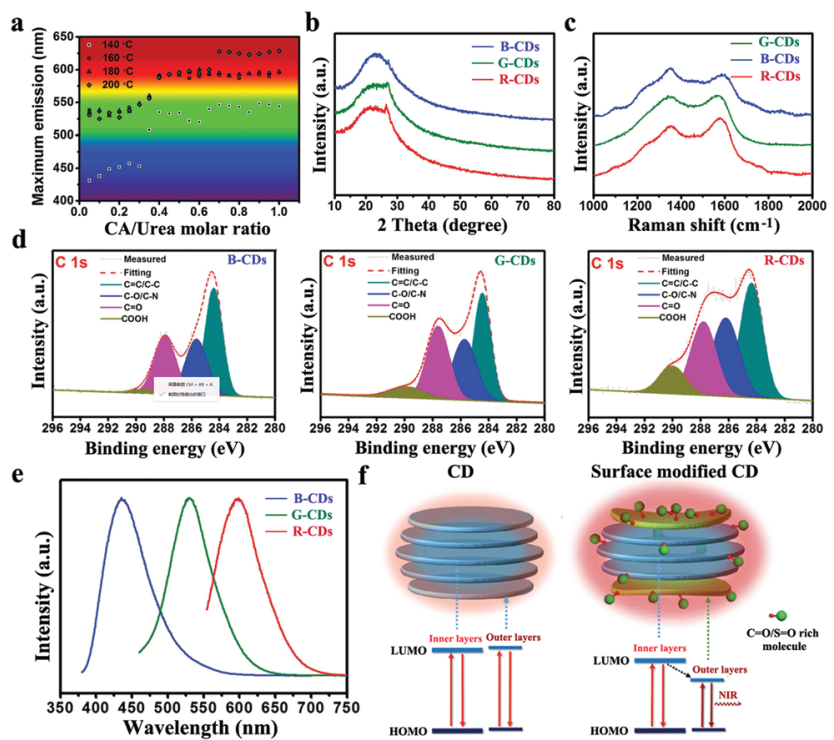

Fig. 2 (a) Maximum emission peaks of CDs at various CA/urea molar ratios and reaction temperatures. XRD patterns (b), Raman spectra (c), high-resolution C1s XPS spectra (d), emission spectra (e) of B-CDs, G-CDs, and R-CDs. Reproduced with permission from ref. 55. Copyright 2018, Wiley-VCH. (f) Schematic of the structure and energy level of CDs and $\mathrm{S}=\mathrm{O} / \mathrm{C}=\mathrm{O}$-rich molecule modified $\mathrm{CD}$. Reproduced with permission from ref. 56. Copyright 2018, Wiley- $\mathrm{VCH}$.
In most cases, PL enhancement with multistep modification is less than 10-fold; processes are often time consuming, requiring toxic chemicals, and unfeasible on a large scale, thereby hindering further imaging applications. ${ }^{57}$ Aggregation-induced emission (AIE) regulates the fluorescent properties of CDs. ${ }^{58-60}$ Recently, $\mathrm{Hu}$ et al. prepared hydrophobic CDs (H-CDs) with excellent AIE properties by using melamine and dithiosalicylic acid/acetic acid (Fig. 3a) ${ }^{61} \mathrm{H}$-CDs display blue fluorescence in a dispersed state, but red emission in an aggregated state by the introduction of water to the solution (Fig. 3b). These interesting properties are ascribed to AIE induced by the $\pi-\pi$ stacking interactions of carbonized cores and the restriction of CD surfaces' intramolecular rotation around disulfide bonds. We also showed that the FL intensity of CDs is enhanced up to 69 times by ionic and nonionic surfactants. The effects of ionic and nonionic surfactants on the FL emission of CDs have been explored for the first time, subsequently improving the sensitivity of CDs for bioimaging. ${ }^{57}$

Compared with visible/NIR-I light-based bioimaging, FL imaging in the NIR-II region (1100-1600 nm) shows substantially reduced scattering losses, enabling the observation of deeper tissues with an enhanced spatial resolution. ${ }^{62-64}$ Hence, nanomaterials

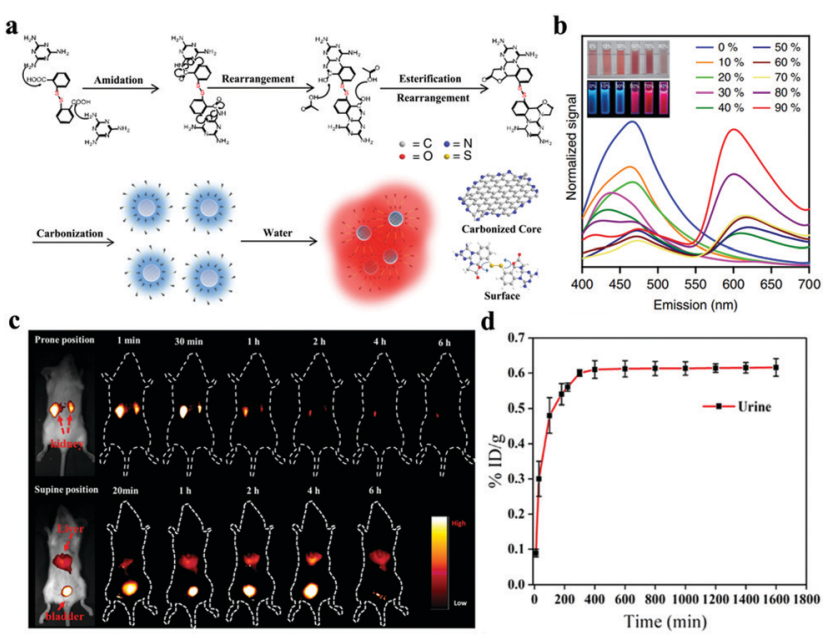

Fig. 3 (a) Mechanism of chemical reactions during the solvothermal treatment of $\mathrm{H}$-CDs. (b) Emission spectrum of $\mathrm{H}$-CDs with different water/ethanol ratios (insets: photographs of $\mathrm{H}-\mathrm{CD}$ solutions under $365 \mathrm{~nm}$ UV light). Reproduced with permission from ref. 61. Copyright 2019, Nature. (c) Time-dependent NIR-II FL imaging of mice treated with i.v. injection of CDs. (d) Cumulative urine excretion curve of CDs. Reproduced with permission from ref. 69. Copyright 2019, American Chemical Society. 
with NIR-II emission should be explored to improve their competitiveness for further biomedical applications, especially bioimaging. Currently, QDs, carbon nanotubes, rare-earthelement-doped nanoparticles, small-molecule-containing NPs, and conjugated polymer NPs have been investigated as NIR-II contrast agents for imaging biological systems. ${ }^{65-68}$ Although some impressive advances in nanoparticle-based NIR-II imaging probes have been achieved, reports on the NIR-II emission of CDs as FL imaging probes remain few. Hao and coworkers first reported a new type of CDs with NIR-II emission by using environment-friendly watermelon as a precursor via a one-step hydrothermal reaction. ${ }^{69}$ The obtained CDs display an emission peak of 900-1200 nm with a QY of $0.4 \%$ under $808 \mathrm{~nm}$ laser irradiation. Then, they performed in vivo NIR-II FL imaging with CDs in the range of 1000-1400 nm. As shown in Fig. 3c, strong NIR-II FL signals are observed in the kidney within 1 min posti.v. CD injection. The signal further enhances at $0.5 \mathrm{~h}$ and decreases after $1 \mathrm{~h}$ of injection. The FL signal in the kidney weakens after $6 \mathrm{~h}$ of injection, indicating the effective excretion of CDs from the body through renal clearance. In a supinepositioned mouse, FL signals in the bladder are intense, whereas signals in the liver are relatively weak. These findings further indicate the primary renal excretion of CDs. The renal clearance of CDs is also evaluated on the basis of emission intensity of CDs in urine. Experimental results reveal that $65 \%$ of CDs can be excreted through urine (Fig. 3d). These results show that the NIR-II emitted CDs with renal excretion are desirable bioimaging probes. Wang's group prepared $\mathrm{N}$ - and B-doped CDs with 3-aminophenylboronic acid monohydrate as a precursor. $^{70}$ N-B-CDs show a broad absorption band at 650-950 $\mathrm{nm}$ and a photoluminescence at 950-1100 $\mathrm{nm}$ with a peak at $1000 \mathrm{~nm}$. They also possess good water solubility, photostability, biocompatibility, and low cytotoxicity. Given these fine optical and physicochemical properties, N-B-CDs are desirable agents for the NIR-II imaging of internal organs and blood vessels.

Two-photon FL imaging (TPFI), which commonly uses NIR femtosecond pulse lasers as light sources, has also received considerable attention in basic biological research and clinical diagnostics because of its large penetration depth, minimized tissue fluorescence imaging with advantages of autofluorescence background, and reduced photodamage in biotissues. ${ }^{71-74}$ To date, various CDs have been widely constructed as two-photon reagents. In 2007, Sun et al. first used surface-passivated CDs with a high two-photon absorption cross-section of 39000 GöppertMayer (GM) units for multiphoton imaging. ${ }^{75}$ Subsequently, Yang and coworkers reported TPFI by using CDs for cell labeling in 2012. ${ }^{76}$ During this period, no studies explored the application of CDs as TPFI probes. In 2013, Gong and coworkers first reported biocompatible N-doped CDs with QY of 0.31 as effective TPFI probes for cellular and deep-tissue imaging. ${ }^{77} \mathrm{~N}$-Doped CDs are synthesized by using dimethylformamide as nitrogen source and solvent. The two-photon absorption cross-section of N-doped CDs reaches 48000 GM, thereby achieving the highest value ever reported for carbon-based nanomaterials and surpassing that of organic dyes. A large penetration depth of $1800 \mu \mathrm{m}$ can be achieved by $\mathrm{N}$-doped CDs in a tissue phantom, significantly

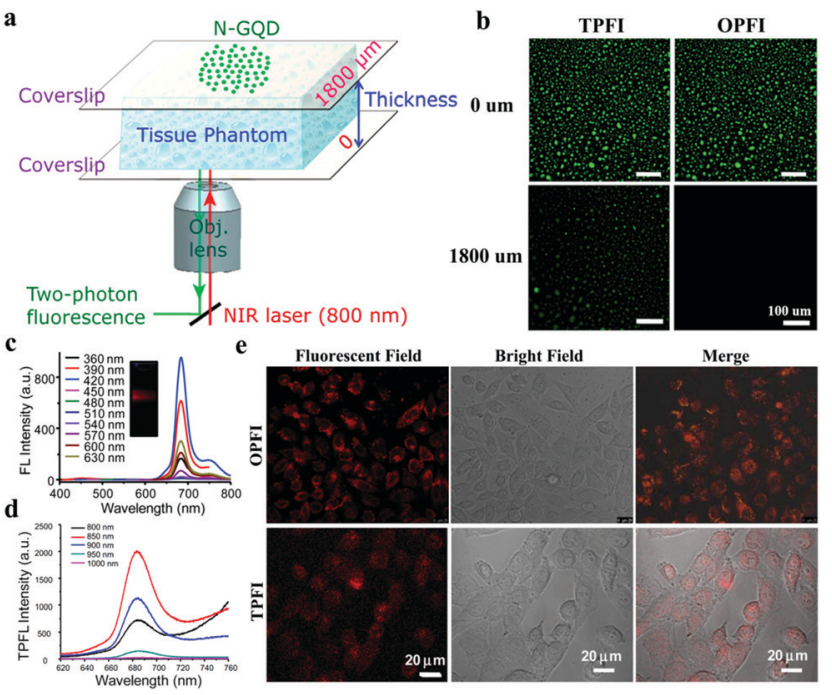

Fig. 4 (a) Schematic of the setup applied for TPFI of N-GQDs in a tissue phantom with varying thicknesses. (b) Penetration depth of N-GQDs for TPFI and OPFI in the setup of (a). Reproduced with permission from ref. 77. Copyright 2013, American Chemical Society. (c) One-photon fluorescence spectra of NIR CDs under different excitation wavelengths. (d) Twophoton fluorescence spectra of NIR CDs with different laser wavelengths of a femtosecond pulse laser. (e) OPFI and TPFI images of NIR CDs in MCF-7 cells. Reproduced with permission from ref. 78. Copyright 2016, the Royal Society of Chemistry.

extending the fundamental TPFI depth limit (Fig. 4a and b). Therefore, N-doped CDs are attractive TPFI probe candidates in biological applications. In 2016, NIR emissive CDs with emission peak at $683 \mathrm{~nm}$ and a high QY of $16.8 \%$ were first reported by Lin's group. ${ }^{78}$ Further investigations have revealed that the emission of NIR CDs is derived predominantly from a surface molecular state mechanism. As shown in Fig. 4c, NIR CDs emit deep-red light with excitation-independent properties. Similar to one-photon fluorescence, excitation-independent emission with $\lambda_{\text {max }} \approx 683 \mathrm{~nm}$ is observed under a femtosecond pulse laser with wavelengths varying from $800 \mathrm{~nm}$ to $1000 \mathrm{~nm}$, with $\sim 850 \mathrm{~nm}$ as the optimum (Fig. 4d). As seen in Fig. 4e, deep-red light images from MCF-7 cells can be achieved under one- and two-photon modes via confocal imaging, suggesting the capabilities of NIR CDs as one-photon FL imaging (OPFI) and TPFI agents. Zhang and Wang reported two-photon excited S, Se-codoped CDs with peaks at 731 and $820 \mathrm{~nm}$ for fluorescence imaging. ${ }^{79}$ Yang and coworkers prepared NIR-emissive polymer carbon nanodots (710 nm, QY 26.28\%) for TPFI. ${ }^{25}$ They were the first to report polymer carbon nanodots with such long-wavelength emission and two-photon fluorescence. Further studies have revealed that a specific $\mathrm{D}-\pi-\mathrm{A}$ structure with a large conjugated rigid $\mathrm{sp}^{2}$ domain may be responsible for a large two-photon absorption cross-section. Qu and Rogach et al. described efficient NIR emissive CDs $\left(\lambda_{\mathrm{abs}} \approx 715-724 \mathrm{~nm}, \lambda_{\mathrm{em}} \approx 750-760 \mathrm{~nm}\right)$ through the surface engineering of molecules or polymers rich in sulfoxide/ carbonyl groups. ${ }^{56}$ They were also the first to present two-photonand three-photon-induced red emission simultaneously observed in CDs. All these works have demonstrated the attractive optical features and promising applications of CDs in the NIR region and 
provided a rational design method for exploiting the two-photoninduced fluorescence of CDs as deep-tissue imaging probes.

\subsection{Carbon dots as photoacoustic imaging probes}

Each imaging modality has unique advantages and intrinsic limitations. Although FL imaging technique shows high sensitivity, spatial resolution is unsatisfactory because of the limited penetration depth. ${ }^{80,81}$ PA imaging, which combines the advantages of ultrasound imaging and optical imaging, is a new emerging bioimaging technique with rich contrast, high resolution, and deep-tissue penetration. ${ }^{82,83}$ This hybrid technique relies on the photoacoustic effect, which refers to the generation of acoustic waves as light converted to heat following optical absorption (Fig. 5a). ${ }^{84}$ Given unusual optical properties, various extrinsic endogenous contrast agents, e.g., oxyhemoglobin and deoxyhemoglobin, and exogenous contrast agents, e.g., organic dyes and nanoparticles, have been employed as PA imaging probes to visualize hierarchical biostructures, including organelles, cells, and organs, especially in cancer diagnosis. ${ }^{85-88}$ Among these agents, CDs have been widely studied as PA imaging probes. In 2015, our group developed a new CD by using polythiophene phenylpropionic acid (PPA), a conjugated polymer synthesized as a precursor in our laboratory. ${ }^{89}$ The as-prepared CDs show a broad absorption band in the visible region to the NIR region (from $400 \mathrm{~nm}$ to $800 \mathrm{~nm}$ with a peak at $470 \mathrm{~nm}$ ) with red emission peaked at $640 \mathrm{~nm}$ with excitation-dependency. The PA spectrum of CDs in an agarose gel phantom reveals obvious

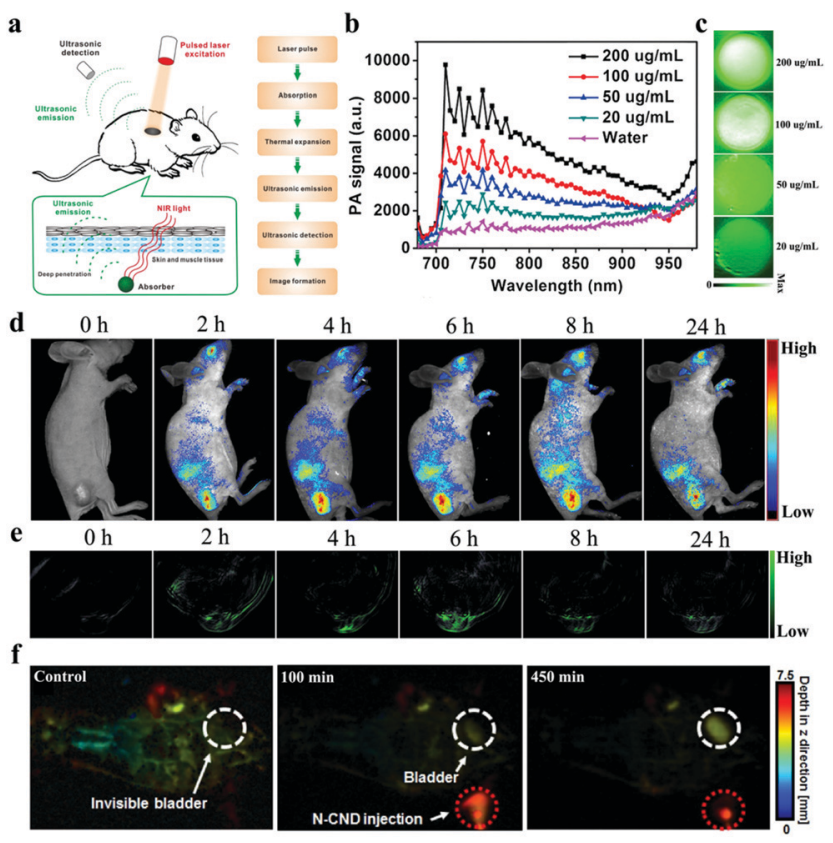

Fig. 5 (a) Schematic of the PA imaging process. Reproduced with permission from ref. 84. Copyright 2016, Ivyspring. PA spectrum (b) and PA imaging (c) of CDs with different concentrations. (d) In vivo FL images of mice after i.v. injection of CDs. (e) PA images of tumors in mice after i.v. injection with CDs. Reproduced with permission from ref. 89. Copyright 2015, Wiley-VCH. (f) In vivo PA imaging of bladder after subcutaneous injection of N-CDs. Reproduced with permission from ref. 90. Copyright 2016, Ivyspring. signals in the range of 700-950 $\mathrm{nm}$ with a peak at approximately $710 \mathrm{~nm}$ (Fig. 5b), and PA signals increase with CD concentration (Fig. 5c). Significantly, red emissive CDs can simultaneously act as FL/PA imaging agents. FL imaging offers high sensitivity and PA imaging provides high-spatial resolution for accurate tumor detection (Fig. 5d and e). We further prepared another CD with Hypocrella bambusae (HB), a parasitic fungus in bamboo, as a raw carbon source via the solvothermal method. ${ }^{91}$ The HBCDs have good water solubility, broad absorption, red emission, and excellent biocompatibility. They can be used as bimodal FL/PA imaging agents to guide tumor therapy. Similarly, a clear tumor microstructure with high spatial resolution and deep penetration is obtained using HBCDs. In comparison with polythiophene-derived CDs, HBCDs are prepared using natural biomass as raw carbon sources, which can effectively resolve problems on complex synthetic routes and high cost. However, these described CDs exhibit weak absorption in the red to NIR region. Qu and his coworkers prepared supra-CDs by using citric acid and urea as carbon sources. ${ }^{92}$ The obtained aqueous supraCD solution exhibits a broad absorption band from $500 \mathrm{~nm}$ to the NIR region with maximum absorption at $670 \mathrm{~nm}$. This result indicates that supra-CDs can be used as an efficient NIR lightresponsive contrast agent for PA imaging at relatively high irradiating power densities $\left(1 \mathrm{~W} \mathrm{~cm}^{-2}\right)$. To enhance the PA signals of CDs in tumors, Wu et al. modified the surface of porphyrin-implanted CDs with cetuximab, which can recognize and target specific cancer cells overexpressing of epidermal growth factor receptor so that they can actively target tumors. ${ }^{93}$ The as-prepared CDs can enhance PA amplitude signals and maintain a strong signal for $12 \mathrm{~h}$ in the bodies of mice. Thus, these CDs can provide a long-term and accurate guidance for the efficient PDT of breast cancer. Kim and coworkers synthesized $\mathrm{N}$-doped CDs with citric acid as a carbon source and nitric acid as a $\mathrm{N}$ source. ${ }^{90} \mathrm{~N}$-CDs have a strong absorption in the NIR region and are responsible for apparent PA signals. Whole-body PA imaging is performed after N-CDs are subcutaneously injected to determine to determine the bodily distribution and clearance behavior. As shown in Fig. 5f, the PA signal in the bladder increases and generally enhances up to $450 \mathrm{~min}$, demonstrating the efficient removal of N-CDs from the body through renal clearance.

\subsection{Carbon dots as magnetic resonance imaging probes}

As a non-invasive imaging mode, MR imaging has been widely used to provide anatomical, physiological, and even molecular information of the body in clinical settings. Given an ability to provide high-contrast images of soft tissues and appropriate penetration depth to visualize the entire human body, MR imaging is often used to verify whether tumors should be surgically removed. ${ }^{94-96}$ To date, two methods have been mainly applied to adopt CDs for MR imaging applications: (1) incorporation of metal ions in CDs and (2) use of metal-free CDs. ${ }^{97,98}$ For example, Chen et al. prepared Gd@GCNs via a solvothermal process as shown in Fig. $6 \mathrm{a}^{94}$ The high resolution transmission electron microscopes (HRTEM) images (Fig. 6b) show that Gd@GCNs are relatively monodispersed and highly crystalline. Element mapping analysis 

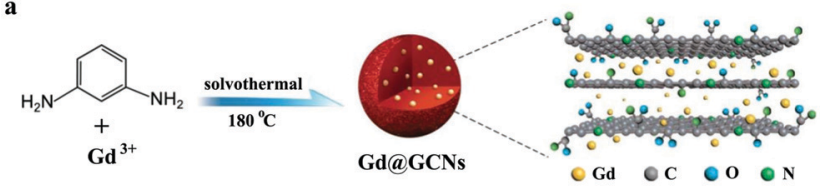

b
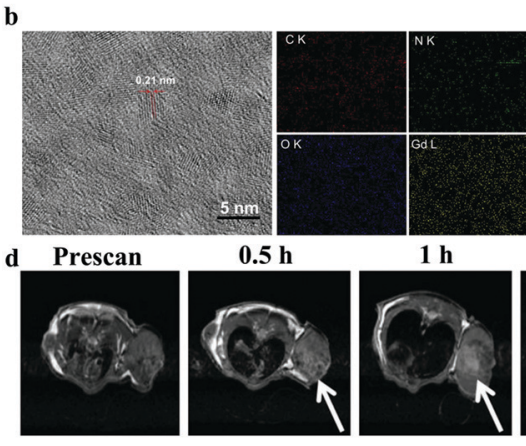

h

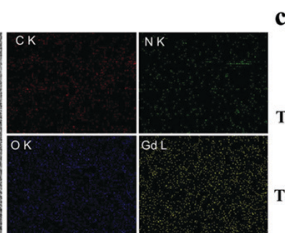

carbon layer

Gd concentration (mM)

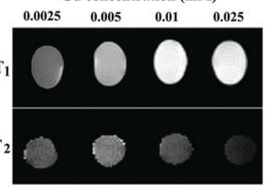

$3 \mathbf{h}$

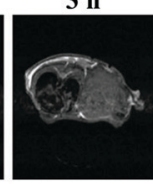

$24 \mathrm{~h}$

Fig. 6 (a) Synthesis of Gd-encapsulated graphene carbon nanotheranostics (Gd@GCNs). (b) HRTEM image and element mapping (C, N, O, and Gd) of GdaGCNs. (c) $T_{1}$ and $T_{2}$ MR images of Gd@GCNs at different $G d$ concentrations. (d) In vivo MR images of the tumor after i.v. injection of Gd@GCNs. Reproduced with permission from ref. 94. Copyright 2018, Wiley-VCH.

also demonstrates that Gd@GCNs have a high Gd content of $37.6 \mathrm{wt} \%$, which provides excellent $T_{1}$ and $T_{2}$ MR imaging effects (Fig. 6c). After i.v. injection of Gd@GCNs into SCC-7 tumor-bearing mouse, the signals on the $T_{1}$-weighted images of tumors significantly enhance upon scanning times on a $7 \mathrm{~T}$ system (Fig. 6d). Fernandez's group also developed new multimodal contrast agents based on CDs. ${ }^{99}$ They used citric acid and $\mathrm{Gd}^{3+}$ and $\mathrm{Yb}^{3+}$ inorganic salts (doping ions) as a precursor to prepare CDs codoped with N, $\mathrm{Gd}$, and $\mathrm{Yb}$, producing a single multimodal contrast agent through a facile and low-cost one-pot microwave-assisted hydrothermal method. The as-prepared CDs exhibit intense and stable FL emission with high QYs (66\% $\pm 7 \%$ ). Moreover, $\mathrm{Gd}^{3+}$ doping can provide CDs with MR contrast capability. The measured $r_{1}$ and $r_{2}$ are 5.02 and $14.45 \mathrm{~mm}^{-1} \mathrm{~s}^{-1}$, respectively, providing $r_{2} / r_{1}$ of 2.88, which indicates that these co-doped CDs are excellent species to be efficiently used as positive $T_{1}$ MR imaging contrast agents. Notably, the longitudinal relaxivity $\left(r_{1}\right)$ of the codoped CDs is greater than that of Magnevist, a commercially available Gd-DTPA contrast agent. Interestingly, the additional incorporation of $\mathrm{Yb}^{3+}$ as a doping ion in CDs can enable them to be CT contrast agents because of the high degree of X-ray absorption of $\mathrm{Yb}^{100}$ The slope of the linear relationship between CT values and $\mathrm{Yb}$ contents $\left(14.4 \mathrm{HU} \mathrm{mm} \mathrm{m}^{-1}\right)$ is higher than that of clinically used iobitridol (i.e., $5.0 \mathrm{HU} \mathrm{mm}^{-1}$ ). This work provides a simple approach for preparing CDs with unique contrast properties for multimodal bioimaging and encourages further exploration for their application in nanobiomedicine.

However, safety concerns limit at the application of Gd or $\mathrm{Yb}$ complexes in renal-compromised patients. Nontoxic $\mathrm{Mn}$ or Fe is induced in CDs as a novel contrast agent for $T_{1}$-weighted MR imaging to overcome this shortcoming. Our group also reported Mn-CDs with a simultaneous bimodal FL/MR imaging characteristic through solvothermal treatment of the precursor
$\mathrm{Mn}$ (II) phthalocyanine at $180{ }^{\circ} \mathrm{C} .{ }^{101}$ The MR signal intensities of Mn-CDs increase with concentration. In vivo $T_{1}$-weighted MR imaging results confirm the high retention rate of Mn-CDs in tumors. Quantitatively, at $6 \mathrm{~h}$ postinjection of Mn-CDs, the MR signal intensity at the tumor site increases by $\sim 320 \%$. By contrast, after the i.v. injection of CDs without doped Mn(II), the MR imaging signal remains nearly unchanged. Jayasree et al. prepared zerovalent iron (ZVI) doped-CDs with a size of approximately $10 \mathrm{~nm} .{ }^{102}$ The obtained ZVI-doped-CDs exhibit a paramagnetic property with $r_{1}$ of $4.93 \mathrm{mM}^{-1} \mathrm{~s}^{-1}$, indicating their capability of being used as a positive MR imaging contrast agent. They further modified ZVI-doped CDs with the livertargeting polysaccharide pullulan and then investigated their capability of being used as dual MR and optical imaging contrast agents for specifically targeting the liver in vivo. Another novel, safe, and efficient $T_{1}$ contrast agent based on $\mathrm{Fe}^{3+} @ \mathrm{~F}, \mathrm{~N}$ codoped-CDs was developed by Bi's group. ${ }^{103}$ $\mathrm{Fe}^{3+} @ \mathrm{~F}, \mathrm{~N}$ codoped-CDs exhibit $r_{1}$ of $5.79 \mathrm{mM}^{-1} \mathrm{~s}^{-1}$, which is higher than those of $\mathrm{Fe}^{3+}$ @CD $\left(4.23 \mathrm{mM}^{-1} \mathrm{~s}^{-1}\right)$ and free $\mathrm{Fe}^{3+}$ (1.59 $\left.\mathrm{mM}^{-1} \mathrm{~s}^{-1}\right)$. Therefore, the codoped CD can be considered new $T_{1}$-weighted MR imaging contrast agents for clinical use because of their biosecurity, high $r_{1}$, strong emission, and cheap synthesis. Novel metal-free CDs, as MR contrast agents, have also been found to act as alternative safe contrast agents for $T_{1}$-weight MR imaging. Zhang and coworkers developed a metal-free boron-doped CQD by using 4-vinylphenylboronic acid and boric acid as precursors (Fig. 7a). ${ }^{104}$ As shown in Fig. 7b, the obtained B-CQDs show an extra-high $r_{1}$ of $18.277 \mathrm{mM}^{-1} \mathrm{~s}^{-1}$, which is greater than that of Gd-CDs $\left(11.35 \mathrm{~mm}^{-1} \mathrm{~s}^{-1}\right) .{ }^{100}$ After subcutaneous injection, B-CQDs with a small diameter enhance the contrast in $T_{1}$-weighted imaging in vivo (Fig. $7 \mathrm{c}$ ). They are promising materials based on CDs for MR imaging in the biological field.

CD chemical exchange saturation transfer (CEST) MR imaging contrast agents have also been reported. Liu and coworkers prepared novel arginine-modified CDs (AC-dots) with abundant exchangeable protons of hydroxyl, amine, and amide anchored

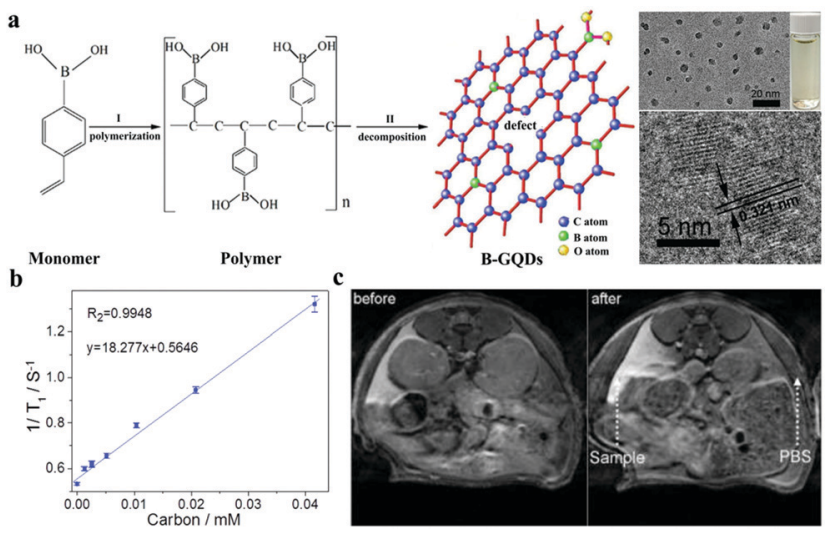

Fig. 7 (a) Schematic of the formation of B-GQDs. (b) Plot of $1 / T_{1}$ as a function of B-GQD concentration. (c) In vivo $T_{1}$-weighted MR images of mice before and after the subcutaneous injection of B-GQDs. Reproduced with permission from ref. 104. Copyright 2017, Wiley-VCH. 


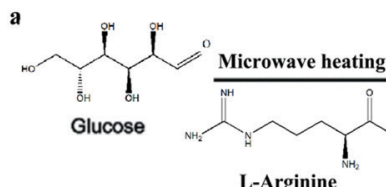

b

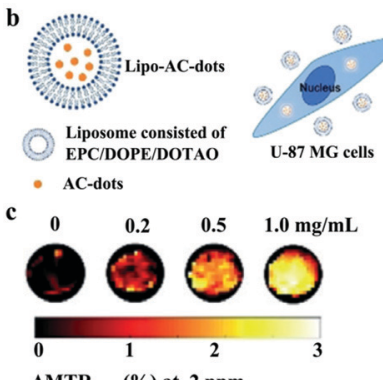

$\triangle \mathrm{MTR}_{\text {asym }}(\%)$ at $2 \mathrm{ppm}$
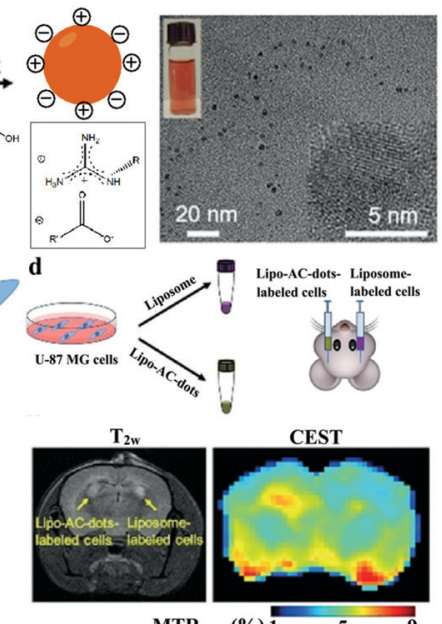

Fig. 8 (a) Synthesis route of AC-dots and surface modification with delocalized Gd cation. (b) Schematic of liposome encapsulated AC-dots for cellular uptake. (c) CEST MR imaging measurements of cells labeled with Lipo-AC-dots. (d) $T_{2}$-Weighted image and the corresponding CEST image of a mouse brain after the implantation of Lipo-AC-dot-labeled cells. Reproduced with permission from ref. 105. Copyright 2019, Wiley$\mathrm{VCH}$.

on the surface for CEST MR imaging without paramagnetic doping (Fig. 8a). ${ }^{105}$ They encapsulated AC-dots with liposomes (Lipo-AC-dots) to increase the cellular uptake of AC-dots (Fig. 8b). As reported, the CEST CDs can be applied as a new type of metal-free MR imaging agents to detect human glioma cells in vitro (Fig. 8c) and in vivo (Fig. 8d) after intracranial implantation. This study can be further extended to design and synthesize CDs with new structures and functions of new CEST MR imaging contrast agents.

\section{Carbon dots for photo-induced cancer treatment}

In early stages, CDs were only used as fluorescent probes to construct FL-imaging-mediated drug delivery by combining them with anticancer drugs for tumor treatment because of their unique luminescent properties. ${ }^{106-108}$ For example, Chen et al. successfully loaded Chlorin e6 (Ce6), a traditional PS, on the surface of CDs and demonstrated that CD-Ce6 is a good candidate with an excellent tumor-homing and PDT ability mediated by NIR FL imaging. ${ }^{109}$ Zheng and coworkers developed a Förster resonance energy transfer (FRET)-based CD-DOX delivery system that can utilize changes in FRET signals generated by the separation distance between CDs and DOX to monitor real-time anticancer drug release under acidic conditions. ${ }^{110}$ To integrate the optical properties of CDs and therapeutic performance of anticancer agents, Sun et al. successfully conjugated oxidized oxaliplatin on the surface of CDs. ${ }^{111}$ In vitro and in vivo results indicate that the fluorescence of CDs can effectively monitor the track or distribution of oxidized oxaliplatin and thus optimize the injection time and dosage of the medicine. Although CDs as drug carriers have achieved good cancer diagnosis and treatment results, some serious problems, such as complex synthetic routes and drug leakage, remain. In addition to ensuring excellent optical properties of CDs, providing themselves with intrinsic therapeutic properties has been widely explored in the biological field. In this section, we review recent reports on CDs with inherent PDT, PTT, and multimodal or stimulus-responsive phototherapeutic properties to clarify their important role in cancer therapy.

\subsection{Carbon dots with intrinsic photodynamic properties}

As a non-invasive treatment triggered by light, PDT has gradually evolved as the fourth-line anticancer method following surgery, chemotherapy, and radiotherapy. ${ }^{112-114}$ In PDT, PSs transfer energy to surrounding oxygen under the excitation of a specific wavelength light to produce ROSs, including ${ }^{1} \mathrm{O}_{2}, \mathrm{O}_{2}{ }^{\bullet-}, \mathrm{H}_{2} \mathrm{O}_{2}$, and ${ }^{\bullet} \mathrm{OH}$, which can strongly kill or cause cancer cell apoptosis. ${ }^{115-117}$ In 2011, Christensen et al. demonstrated that CDs with a surface passivated by polyethylene glycol can produce ROS upon irradiation with blue light. ${ }^{118}$ In 2012, Markovic and coworkers were the first to prove the photodynamic cytotoxicity of CDs toward cancer cells. ${ }^{119}$ Since then, CDs with intrinsic PDT properties have attracted considerable attention. In 2014, our group developed a new PDT agent based on CDs with NIR emission by using PT2 as a carbon source. ${ }^{39}$ The $^{1} \mathrm{O}_{2}$ yield of CDs is as high as 1.3. The super high ${ }^{1} \mathrm{O}_{2}$ yield of CDs is attributed to two pathways (Fig. 9a): one is the energy transfer from the path from the $\mathrm{T}_{1}$ state to the $\mathrm{S}_{0}$ state via traditional PSs (the ${ }^{1} \mathrm{O}_{2}$ yield is always less than 1.0), and the other occurs from the $S_{1}$ state to the $\mathrm{T}_{1}$ intersystem crossing transition. Therefore, the superposition of these two approaches can ensure that the ${ }^{1} \mathrm{O}_{2}$ yield of CDs is greater than 1.0. These excellent ${ }^{1} \mathrm{O}_{2}$ properties of CDs enable them to achieve an effective PDT of cancer in vitro (Fig. 9b) and in vivo (Fig. 9c). However, this CD PS has low therapeutic efficacies for deep tumor tissues due to its absorption in the visible

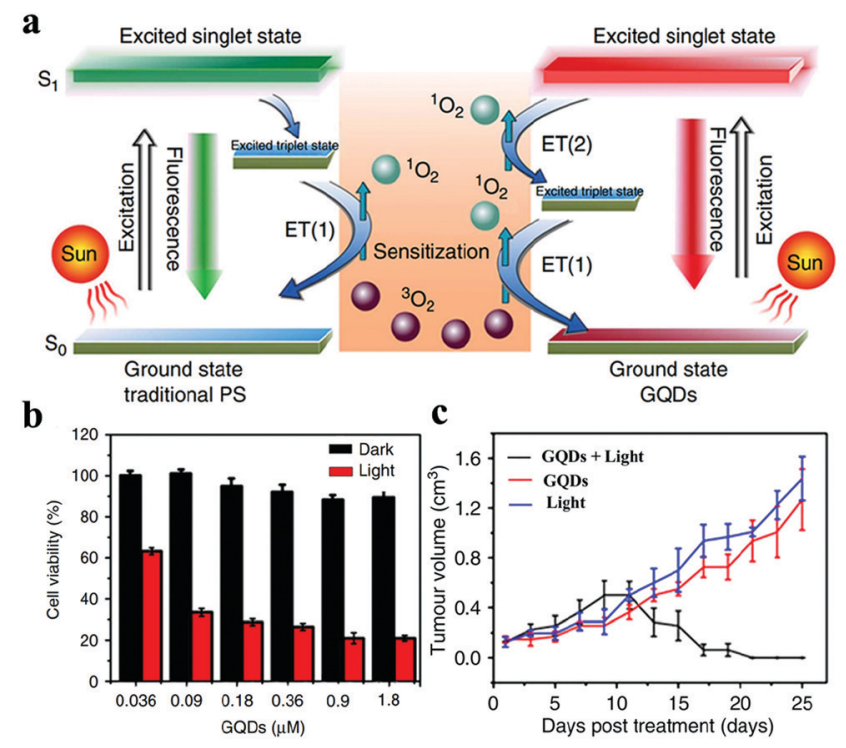

Fig. 9 (a) Schematic of the ${ }^{1} \mathrm{O}_{2}$ generation by classical PSs (left) and GQDs (right). (b) In vitro PDT effects of HeLa cells treated with GQDs under light irradiation. (c) Growth curves of tumors treated with GQDs under light irradiation. Reproduced with permission from ref. 39. Copyright 2014, Nature. 
light region. In addition, the positive charges of CD surface produce undesirable effects in vivo via i.v. administration, consequently resulting in mouse death. ${ }^{120}$ To address these limitations, our group prepared an NIR-light-responsive CD nanosphere (CDNS) via self-assembly by using individual CDs as building units. ${ }^{121}$ In comparison with individual CDs, the assembled CDNSs exhibit a red-shifted absorption of $\sim 50 \mathrm{~nm}$, a broader absorption band from $400 \mathrm{~nm}$ to $800 \mathrm{~nm}$, and a stronger NIR emission peak at $730 \mathrm{~nm}$. The positively charged surface of $\mathrm{CD}$ changes into the negatively charged surface of CDNSs. CDNSs can efficiently generate ${ }^{1} \mathrm{O}_{2}$ with a high QY of $45.4 \%$ under NIR laser irradiation. Thus, NIRlight-responsive FL-imaging-guided PDT can be simultaneously performed after i.v. administration in vivo. To overcome difficulties in synthesizing CDs with an intrinsic PDT activity, Zhu et al. imbedded various metals [M = Zn(II) or Mn(II)] or metal-free tetra(meso-aminophenyl)porphyrin into CDs during carbonization. ${ }^{122}$ They experimentally demonstrated that the as-prepared CDs have excellent characteristics, such as NIR emission, good water solubility, and favorable biocompatibility, which are observed in porphyrins and pristine CDs. In vitro results show that these porphyrin-embedded CDs exhibit a remarkable PDT efficacy against tumor cells upon irradiation. Tan's group reported fluorescent CDs with the four basic nucleotides of DNA as precursors. ${ }^{123}$ They compared four kinds of CDs and found that adenosine triphosphate-CDs show the highest fluorescence QY of $13.9 \%$, adenosine diphosphate-CDs exhibit the best photostability that maintains $97.6 \%$ fluorescence intensity after 30 min of UV illumination, and deoxyadenosine monophosphate (dAMP)-CDs have the highest ${ }^{1} \mathrm{O}_{2}$ QY of 1.20. dAMP-CDs have a good cell absorption ability indicated by their fluorescence and efficacy of killing cancer cells in vitro under white light treatment.

CDs with PDT functions have been rarely reported because of a complex preparation route and difficulties in choosing carbon sources. A simple and straightforward strategy to prepare PDT CDs has been an emerging focus. For example, Xie et al. prepared a porphyrin-containing CD (triphenylporphyrin (TPP) CD) and demonstrated its effective photodynamic activity in tumor treatment. ${ }^{124}$ As shown in Fig. 10a, TPP CDs are synthesized from monohydroxylphenyl TPP and chitosan. After hydrothermal treatment at $180{ }^{\circ} \mathrm{C}$ for $20 \mathrm{~h}$, the generated TPP CDs retain the aromatic structure of TPP. As a result, TPP CDs gain photodynamic properties. In addition, diketopyrrolopyrrolederived CDs (DPP CDs) prepared through hydrothermal method (Fig. 10b) maintain the ability of DPP to generate ${ }^{1} \mathrm{O}_{2} \cdot{ }^{125}$ Moreover, green carbon sources (e.g., natural biomass) are good candidates for the preparation of CDs with intrinsic PDT properties. Our group developed a kind of CDs with the natural biomass of pheophytin as a raw carbon source via a microwave method (Fig. 10c). ${ }^{126}$ The obtained hydrophobic CDs exhibit an NIR emission peak at $680 \mathrm{~nm}$ and a high ${ }^{1} \mathrm{O}_{2}$ yield with a QY of 0.62. After the assembly with DSPE-mPEG2000, CD assemblies are used not only as an efficient FL imaging agent but also as a smart PDT agent in vitro and in vivo. Mobin and coworkers prepared nitrogen-doped CDs (N@VRCDs) by using ethylenediamine as a nitrogen source and Vigna radiata sprouts as a sole carbon

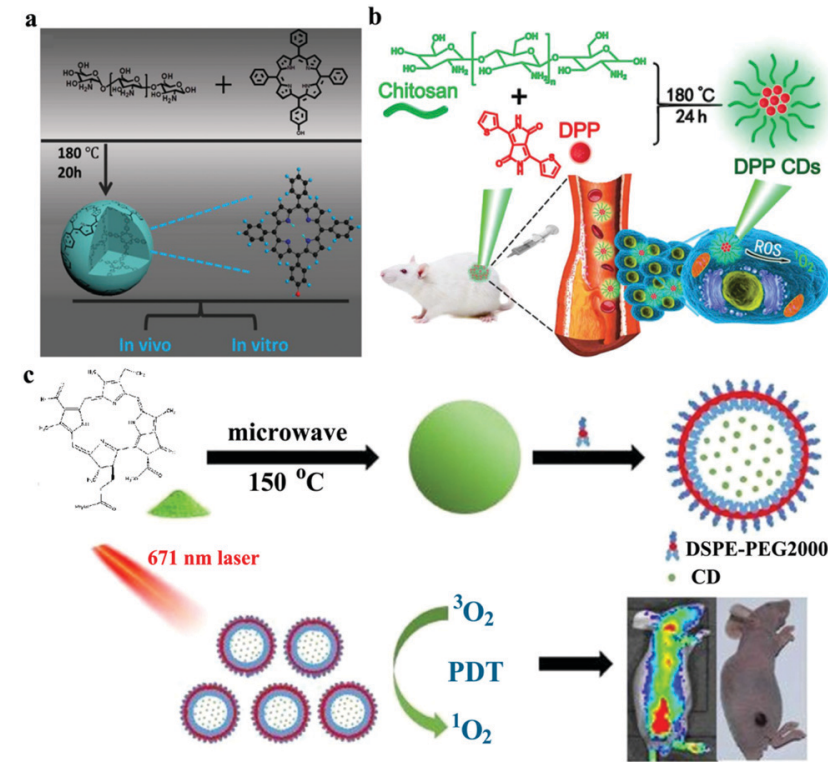

Fig. 10 (a) Synthetic route of TPP CDs. Reproduced with permission from ref. 124. Copyright 2017, Wiley-VCH. (b) Schematic of DPP CDs for the PDT of cancer. Reproduced with permission from ref. 125. Copyright 2018, the Royal Society of Chemistry. (c) Schematic of a pheophytin-derived CD assembly for FL imaging and PDT. Reproduced with permission from ref. 126. Copyright 2019, Wiley-VCH.

precursor. ${ }^{127} \mathrm{~N} @$ VRCDs also exhibits remarkable light-induced cytotoxicity to cancer cells at an ${ }^{1} \mathrm{O}_{2}$ QY of 0.41 . Thus, they may be used as a potential theranostic agent.

As a consequence of the common operation of the type II pathway, most existing PDT systems exhibit high $\mathrm{O}_{2}$ dependence and involve a dramatic consumption of $\mathrm{O}_{2}$. Unfortunately, aggressive cancer cell proliferation and insufficient blood supply lead to low $\mathrm{O}_{2}$ concentrations in the tumor interior, thereby causing a significantly reduced PDT efficacy. ${ }^{128}$ In contrast to type II route, the type I PDT takes place by either electron or hydrogen atom abstraction via excited PS from substrates. This process produces respective radical ions or radicals, and type I PDT has been shown to be effective even under low $\mathrm{O}_{2}$ conditions. Therefore, the preparation of CDs for type I PDT may be a new approach to Wang and coworkers prepared reduced graphene oxide quantum dots (rGOQDs) via a simple sonicationassisted thermal exfoliation of graphite powders followed by the reduction of hydrazine hydrate at $90{ }^{\circ} \mathrm{C}$ (Fig. 11a). ${ }^{129}$ The obtained rGOQDs under white light can effectively produce a variety of ROS, including ${ }^{1} \mathrm{O}_{2}, \mathrm{H}_{2} \mathrm{O}_{2}$, and $\mathrm{O}_{2}{ }^{\bullet-}$. Furthermore, the yield of ROS from rGOQDs is about two times that from GOQDS because the reduction of GOQDs with hydrazine hydrate decreases the band gap and valence band of GOQDs and results in the production of more electron hole pairs. Shen et al. reported hyaluronic acid-derived CDs (HA-CDs) that can produce $\mathrm{O}_{2}{ }^{\bullet-}$ instead of ${ }^{1} \mathrm{O}_{2}$ for PDT (Fig. 11b) ${ }^{130}$ Given the large amount of HA remaining on the surface, the as-prepared HA-CDs can actively target CD44-receptor-overexpressing cancer cells. They demonstrated the effective generation of $\mathrm{O}_{2}{ }^{\bullet-}$ from ESR measurements (Fig. 11c). Meanwhile, they mainly attributed 


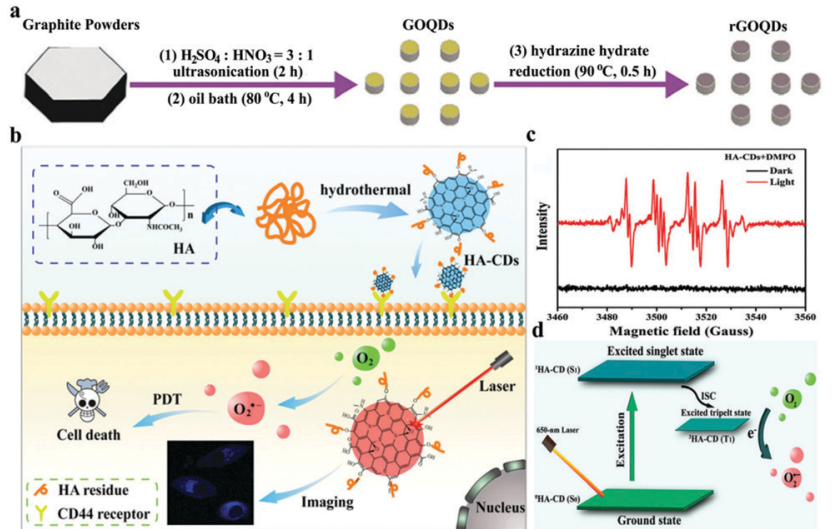

Fig. 11 (a) Schematic of the preparation of GOQDs and rGOQDs. Reproduced with permission from ref. 129. Copyright 2018, the Royal Society of Chemistry. (b) Schematic of HA-CD preparation and the self-targeted imaging-guided PDT of cancer cells. (c) ESR spectra of HA-CDs + DMPO with laser irradiation. (d) Schematic of photo-induced $\mathrm{O}_{2}{ }^{--}$generation by HA-CDs. Reproduced with permission from ref. 130. Copyright 2018, the Royal Society of Chemistry.

$\mathrm{O}_{2}{ }^{\bullet-}$ generation to the photo-induced electron transfer from the excited triplet state of HA-CDs to $\mathrm{O}_{2}$ as shown in Fig. 11d.

\subsection{Carbon dots with intrinsic photothermal properties}

As another mode of phototherapy for cancer, PTT induces the death of cancer cells through photothermal agents harvest light energy and then convert to heat for increasing the environment temperature. ${ }^{131-134}$ In 2015, our group developed a CD PTT agent by using PPA as a precursor (Fig. 12a). ${ }^{89}$ CDs exhibit an excellent photothermal effect with a high conversion efficiency of $38.5 \%$ (Fig. 12b); thus, they can be simultaneously used for FL/PA-imaging (as described in Section 2.2)-guided PTT of

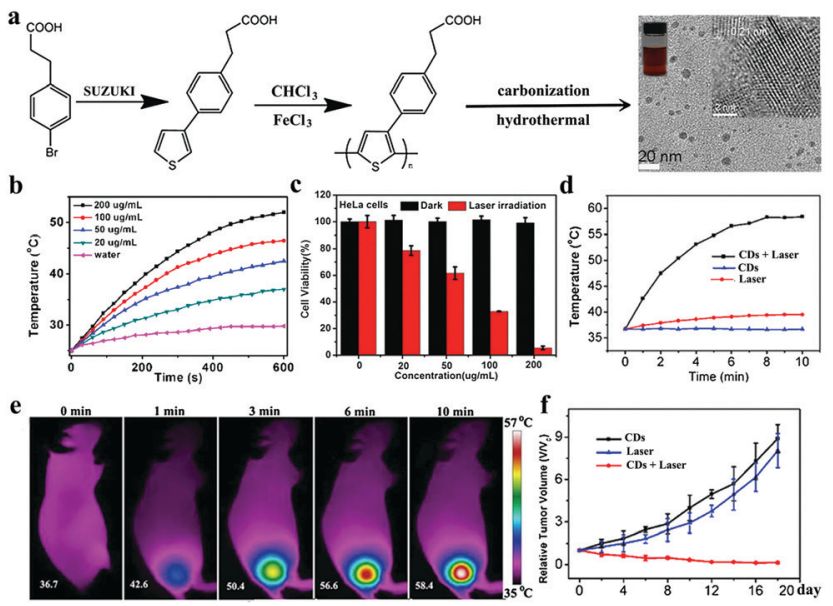

Fig. 12 (a) Synthetic route of PPA-derived CDs. (b) Temperature increase in CDs with varying concentrations as a function of irradiation time (671 nm laser, $2 \mathrm{~W} \mathrm{~cm}^{-2}$ ). (c) Relative viability of HeLa cells incubated with CDs before and after irradiation by $671 \mathrm{~nm}$ laser. (d) In vivo thermal imaging of tumor sites irradiated by $671 \mathrm{~nm}$ laser $\left(2 \mathrm{~W} \mathrm{~cm}^{-2}\right)$ at $6 \mathrm{~h}$ after i.v. injection of CDs. (e) Temperature change curves of tumor sites as a function of irradiation time. (f) Relative growth curves of tumor after different treatments. Reproduced with permission from ref. 89. Copyright 2015, Wiley$\mathrm{VCH}$. cancer in living mice. As shown in Fig. 12c, under $671 \mathrm{~nm}$ laser irradiation $\left(2.0 \mathrm{~W} \mathrm{~cm}^{-2}\right)$, the viabilities of HeLa cells decrease significantly as the concentration of CDs increases, and approximately $100 \%$ mortality rate is achieved at $200 \mu \mathrm{g} \mathrm{mL}$ CDs. When treated with CDs following with NIR laser illumination, the temperature of tumor is higher than $60{ }^{\circ} \mathrm{C}$ (Fig. $12 \mathrm{~d}$ and e). By comparison, the maximum temperature of tumor in the laser-only group is approximately $39.5{ }^{\circ} \mathrm{C}$, indicating the key role of CDs in heat generation. Therefore, CDs significantly suppress tumor growth under NIR laser irradiation as shown in Fig. 12f. Since then, the use of CDs as PTT agents for cancer treatment has provided a basis for further developing these materials. For example, Lin et al. reported a red emissive CD (R-CD) PTT agent with citric acid as a carbon source and formamide as a solvent, respectively. ${ }^{44}$ They pointed out that formamide plays an important role in the preparation of R-CDs because it or its decomposed products participate in R-CD formation. R-CDs exhibit a high photothermal conversion efficiency of $43.9 \%$ under the irradiation of $671 \mathrm{~nm}$ laser, ensuring their excellent photothermal effects against cancer cells in vitro. Qu and coworkers prepared $\mathrm{N}$ - and S-codoped NIR-emitting CDs with citric acid as a carbon source, urea as a nitrogen-doping source, and dimethylsulfoxide as a solvent and sulfur-doping source. ${ }^{135}$ The as-prepared CDs show a broad absorption band with maximum absorption at $600 \mathrm{~nm}$, a strong NIR emission peak at $720 \mathrm{~nm}$, and a high photothermal conversion efficiency (59.19\%, $655 \mathrm{~nm}$ laser). This work demonstrates that the developed CDs are highly efficient in the PTT of cancer in mouse models and can be simultaneously visualized via bimodal FL/PA imaging. They also used previously reported humidity-induced CD assemblies (supra-CDs) as contrast agents for PA imaging and photothermal agents for PTT. ${ }^{92}$ After i.v. injection, supra-CDs can accumulate in tumor tissues as revealed by in vivo PA imaging and effectively inhibit tumor growth under $655 \mathrm{~nm}$ laser irradiation.

The abovementioned CDs are mostly activated by different red lights, but their shallow tissue penetration leads to poor PTT effects. Studies have also explored NIR CDs that can absorb NIR light to produce heat for the treatment of deep tumors (Table 2). In Section 2.2, N-CDs synthesized by Kim et al. using citric acid and nitric acid can be applied not only as a PA imaging contrast agent to assess their renal clearance after hypodermic injection but also as an NIR PTT agent to the photothermal ablation of tumors with N-CDs injected near a tumor region followed by irradiation with $808 \mathrm{~nm}$ laser. ${ }^{90}$ The mentioned NIR-II CDs with watermelon as a precursor also exhibit a high photothermal efficiency of $30.6 \%$ under $808 \mathrm{~nm}$ laser irradiation and show strong potential for the thermal ablation of cancer. ${ }^{69}$ In addition, Pan and coworkers reported a nitrogen and oxygen codoped CD (N-O-CD) from 1,3,6-trinitropyrene and polyethylenimine as shown in Fig. 13a. ${ }^{136}$ Black N-O-CDs exhibit a strong optical absorbance in the NIR region, ensuring their high photothermal conversion efficiency of $38.3 \%$ under the irradiation of $808 \mathrm{~nm}$ laser. In vivo PTT experiments demonstrate that an intensive penetration depth of $808 \mathrm{~nm}$ laser and high-efficiency 
Table 2 NIR light-induced CDs prepared from different carbon sources and their photothermal properties

\begin{tabular}{|c|c|c|c|c|c|}
\hline Carbon source & Method & Doping & $\begin{array}{l}\text { Excitation } \\
\text { wavelength }(\mathrm{nm})\end{array}$ & $\begin{array}{l}\text { Photothermal } \\
\text { conversion efficiency (\%) }\end{array}$ & Ref. \\
\hline Citric acid, nitric acid & Solvothermal & $\mathrm{N}$ & 808 & - & 90 \\
\hline Watermelon juice & Hydrothermal & $\mathrm{N}$ & 808 & 30.6 & 69 \\
\hline Cyanine dye, poly(ethylene glycol) & Solvothermal & $\mathrm{O}$ & 808 & 38.7 & 137 \\
\hline F127, phenol, formaldehyde & Solid-state transformation & $\mathrm{N}, \mathrm{O}$ & 808 & 42.3 & 138 \\
\hline Nigrosin, manganese acetate & Hydrothermal & $\mathrm{Mn}, \mathrm{N}$ & 808 & 7.6 & 139 \\
\hline
\end{tabular}

heat generation achieve an excellent therapeutic efficacy with $100 \%$ tumor destruction. Xie et al. prepared an NIR CD PTT agent (CyCD) from a hydrophobic cyanine dye $\mathrm{CyOH}$ and poly(ethylene glycol) (Fig. 13b). ${ }^{137}$ The as-prepared CyCDs possess NIR emission from $600 \mathrm{~nm}$ to $900 \mathrm{~nm}$ and show a high photothermal conversion efficiency of $38.7 \%$; thus, the as-prepared CyCDs act as ideal nanotheranostic agents for NIR FL imaging and PTT in vitro and in vivo. Huang's group also synthesized highly crystalline CDs (HCCDs) with tunable full-color emissions, a strong PA imaging effect, and a high photothermal ability. ${ }^{138}$ As shown in Fig. 13c, HCCDs are prepared via in situ solid-state transformation with freeze-dried mesoporous phenolic resin generated from F127, phenol, and formaldehyde at $350{ }^{\circ} \mathrm{C}$ with $\mathrm{NaCl} / \mathrm{LiCl} / \mathrm{KNO}_{3}$ as catalyst. High temperature prompts the high crystallization of carbon cores, which are responsible for strong photothermal/ photoacoustic effects. Therefore, HCCDs have been successfully used for the sensitive FL imaging and deep space-resolved PA imaging-guided PTT of glioma. In addition to nonmetallic elements, some metal elements (e.g., Zn and Gd) are doped in CDs to improve their photophysical and photochemical properties. $\mathrm{Mn}$ is one of the essential trace elements and important to human health. It has a multivalent state, which is conducive to conducting a valence state process and improving the optical properties. Cai et al. doped Mn into nigrosin-originated CDs (Mn-CDs). ${ }^{139}$ In comparison with nigrosin-originated CDs, the as-prepared Mn-CDs possess wide absorbance, strong fluorescence emission, and excellent photothermal effects under NIR light. After the i.v. injection of Mn-CDs, in vivo FL/PA imaging results reveal that Mn-CDs can passively target tumor tissues via the enhanced permeation and retention (EPR) effect. When specimens are exposed to $808 \mathrm{~nm}$ laser light, the temperature of tumor tissues with Mn-CD accumulation can increase rapidly to achieve the goal of PTT. In addition to fluorescence emission, the TPE mechanism is applicable to PTT to overcome the shallow tissue penetration of exciting light. Lan et al.

a

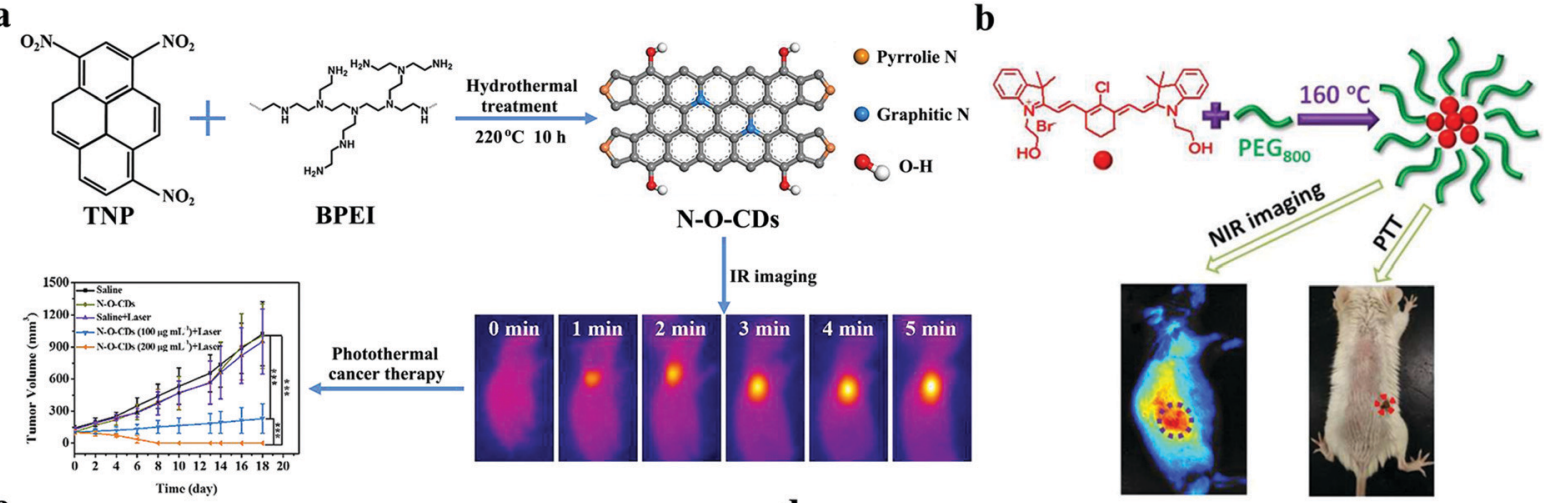

c
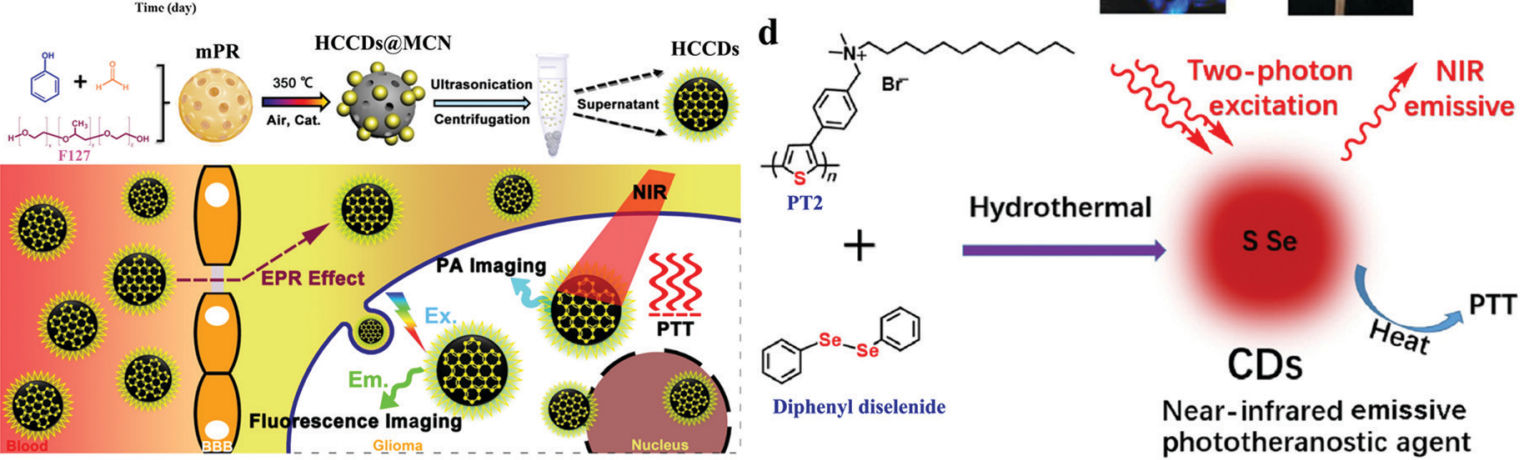

Hydrothermal
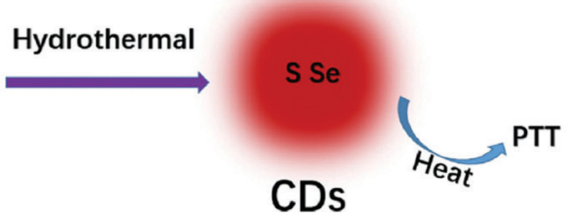

Near-infrared emissive phototheranostic agent

Fig. 13 Synthetic procedure and working mechanism of different CDs, including N-O-CDs (a), with intrinsic PTT properties. Reproduced with permission from ref. 131. Copyright 2018, Elsevier. CyCDs (b). Reproduced with permission from ref. 132. Copyright 2016, American Chemical Society. HCCDs (c). Reproduced with permission from ref. 133. Copyright 2018, American Chemical Society and S- and Se-codoped CDs (d). Reproduced with permission from ref. 73. Copyright 2017, Springer. 
prepared a S- and Se-codoped CD with intrinsic TPE-induced fluorescence and photothermal properties by using a polythiophene derivative and diphenyl diselenide. ${ }^{79}$ As shown in Fig. 13d, S-Se-CD can be simultaneously used for the two-photon-excited FL-imagingguided NIR PTT of cancer. Thus, they can be used as a basis for promoting the biomedical application of CDs as phototheranostic agents.

\subsection{Smart carbon dots for multimodal cancer therapy}

Although PDT and PTT have some anticancer treatment advantages, such as low toxic side effects, noninvasive or minimally invasive treatment, and no drug resistance, each treatment suffers from inherent limitations. For example, PDT is generally oxygen dependent. However, a tumor microenvironment (TME) is usually hypoxic, ${ }^{140}$ which can severely hinder the therapeutic efficacy of PDT. ${ }^{141-143}$ In addition, PTT usually requires high laser power, and the generated hyperthermia inevitably damages normal tissues around tumors. Considerable effort has been devoted to developing multimodal treatment that integrates the advantages of each technique to meet high therapeutic requirements and overcome the inherent limitations of each phototherapeutic technique. ${ }^{144-146}$ Some well-designed nanoplatform-based CDs with intrinsic theranostic properties are available for multimodal cancer treatment. In 2016, our group designed a multifunctional nanoplatform of gold nanorod@silica-CDs (GNR@SiO $\left.{ }_{2}-\mathrm{CDs}\right)$ as phototheranostic agents (Fig. 14a). ${ }^{147}$ In GNR@SiO ${ }_{2}$-CDs, GNRs act as PA imaging and PTT agents with $808 \mathrm{~nm}$ laser, and CDs serve as FL imaging and PDT agents with $635 \mathrm{~nm}$ laser. The high sensitivity and good spatial resolution of the bimodal FL/PA images of tumor demonstrate that GNR@SiO ${ }_{2}$-CDs can gradually accumulate in tumors through the EPR effect after i.v. administration. Thus, this technique provides valuable information for the follow-up light-induced treatment of tumors. Furthermore, the damage induced by the synergy of PDT and PTT to cancer cells is more severe than that caused by either PDT or PTT alone. However, synergistic PTT/PDT utilizes two light sources to separately induce PDT and PTT, and problems on high laser power for PTT remain unsolved. Lin and coworkers loaded PS Ce6 onto amino-rich red emissive CDs (Ce6-RCDs) for synergistic PTT/PDT against cancer triggered by a single $671 \mathrm{~nm}$ laser (Fig. 14b). ${ }^{148}$ Ce6-RCDs provide remarkably higher anticancer effect upon a low power density of laser $\left(0.50 \mathrm{~W} \mathrm{~cm}^{-2}\right)$ than the equivalent Ce6 or RCDs under the same irradiation conditions. Ce6-RCDs also show FL/PA bimodal imaging properties, which can be employed for guidance during phototherapy. Mauro et al. decorated an NIR CD PTT agent (CDs-PEG-BT@IT) with the targeting molecule biotin and the anticancer drug irinotecan to actively recognize cancer cells (Fig. 14c). ${ }^{149}$ CD-PEG-BT@IT can be used as nanoheaters that can trigger local hyperthermia and massive chemotherapeutic drug release inside tumors. Thus, these substances can efficiently provoke tumor death.

In comparison with the abovementioned multimodal therapeutic nanoplatforms prepared using CDs with intrinsic single phototherapeutic properties as carriers, CDs with intrinsic multimodal therapeutic properties have the advantages of optimizing preparation steps, requiring low cost, and preventing drug leakage. In 2016, our group prepared NIR CDs with redlight emission from $640 \mathrm{~nm}$ to $680 \mathrm{~nm}$ by using polythiophene a

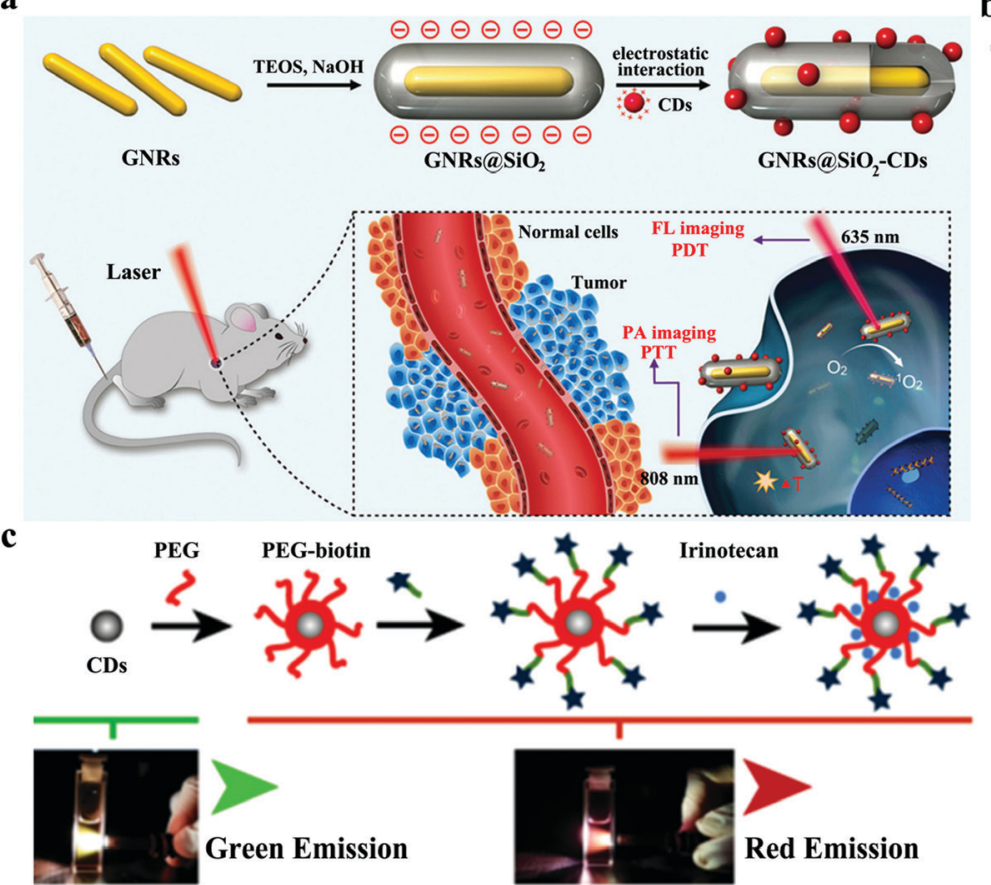

b
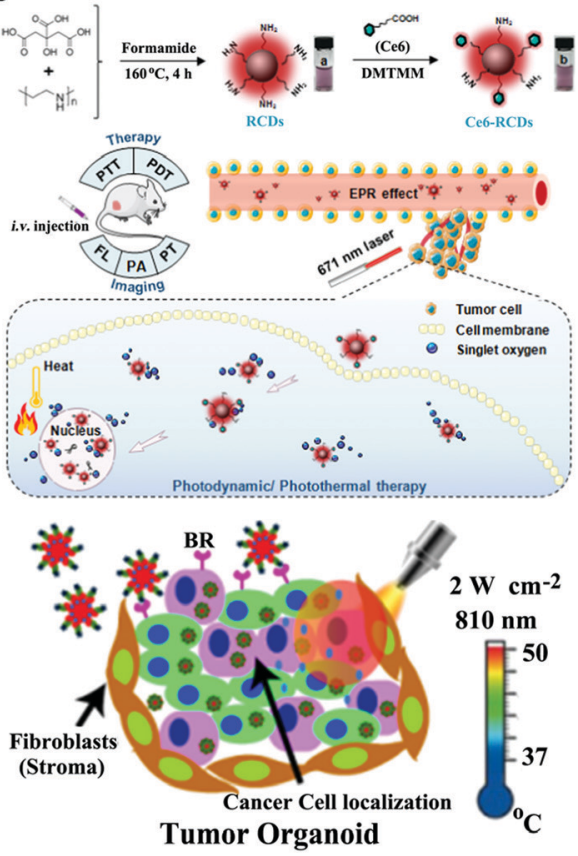

Fig. 14 Mechanism of different CD-based nanoplatforms, including $\mathrm{GNR}_{\mathrm{SSiO}}-\mathrm{CDs}$, for multimodal anticancer therapy (a). Reproduced with permission from ref. 147. Copyright 2016, the Royal Society of Chemistry. Ce6-RCDs (b). Reproduced with permission from ref. 148. Copyright 2019, American Chemical Society. CD-PEG-BT@IT (c). Reproduced with permission from ref. 149. Copyright 2019, American Chemical Society. 


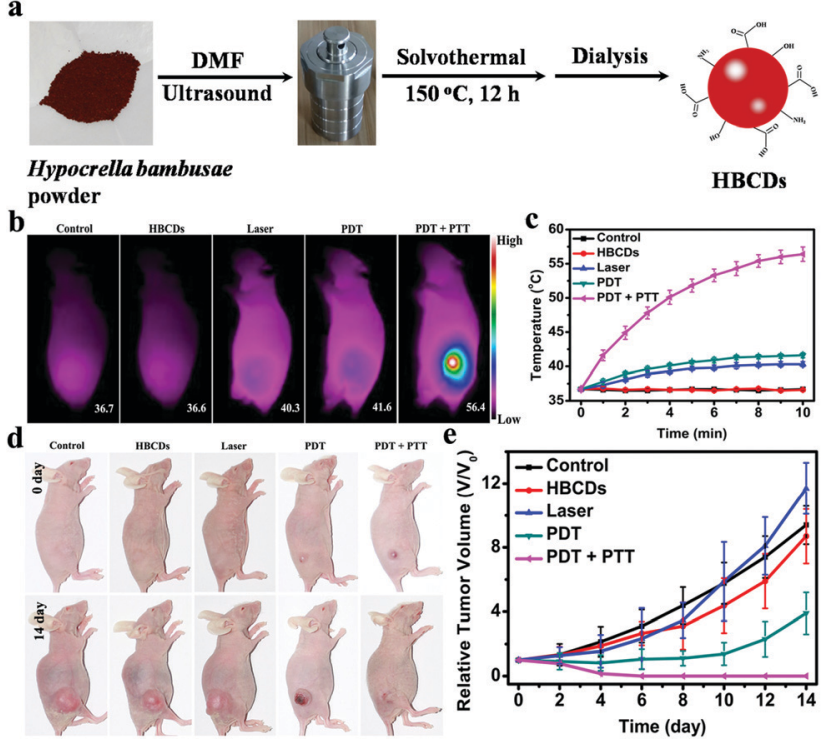

Fig. 15 (a) Synthesis of HBCDs derived from HB. (b) IR thermal images of mice treated with HBCDs and laser. (c) Temperature curves of tumors during irradiation. (d) Images of mice after they received different treatments. (e) Growth curves of tumors treated with HBCDs and laser Reproduced with permission from ref. 91. Copyright 2018, Elsevier.

benzoic acid. ${ }^{150}$ The CDs show dual PDT and PTT effects with an ${ }^{1} \mathrm{O}_{2}$ QY of $27 \%$ and a photothermal conversion efficiency of $36.2 \%$. These excellent characters of CDs enable themselves to be used not only as red FL imaging probes but also as efficient PDT/PTT therapeutic agents. This study is the first to prove the utilization of CDs for the imaging-guided synergistic PDT/PTT in vivo by using a single laser. The HBCDs we prepared can also be used for synergistic PDT/PTT (Fig. 15a). ${ }^{91}$ HBCDs can effectively produce ${ }^{1} \mathrm{O}_{2}(0.38)$ and heat (27.6\%) under $635 \mathrm{~nm}$ laser irradiation. As shown in Fig. 15b and c, when tumor tissues are illuminated with $635 \mathrm{~nm}$ laser $\left(0.8 \mathrm{~W} \mathrm{~cm}^{-2}\right)$ after the i.v. injection of HBCDs, the temperature of tumor tissues rapidly increases to $56.4{ }^{\circ} \mathrm{C}$, which can sufficiently induce severe photothermal damage to cancer cells. Although the growth of the tumors of mice treated with single PDT $\left(635 \mathrm{~nm}, 0.1 \mathrm{~W} \mathrm{~cm}^{-2}\right)$ is efficiently inhibited, it cannot be completely eliminated. By contrast, the growth of the tumors of mice treated with synergistic PDT/PTT is entirely inhibited without recurrence after 14 days of treatment (Fig. 15d and e). These results indicate that the treatment efficacy of the synergistic PDT/PTT modality is better than that of the single PDT. Furthermore, Jiang and coworkers used $\mathrm{Cu}$ (II) to engineer the structural characteristics of CDs and obtain NIR absorption for heat and ROS production. ${ }^{151}$ The as-prepared Cu-CDs can inhibit cancer growth via synergistic PTT/PDT. In addition, Cu-CDs can act as FL imaging and IR thermal imaging agents to visualize in vitro and in vivo treatments, respectively.

\subsection{Tumor microenvironment stimulus-responsive CDs-based nanotheranostic}

A TME is a complex integrated system composed of tumor cells, immune and inflammatory cells, tumor-related fibroblasts, adjacent interstitial tissues, microvessels, and various cytokines and chemokines. ${ }^{152,153}$ In comparison with normal tissues, the TME has unique properties, such as overexpressed biomarkers, hypoxia, mild acidity, high glutathione (GSH) and $\mathrm{H}_{2} \mathrm{O}_{2}$, can reduce the anti-cancer effectiveness of phototherapy. ${ }^{154-156}$ However, the TME has positive and negative effects. TME features can provide opportunities for preparing stimulus-responsive phototheranostic nanoplatforms to achieve efficient and less toxic tumor treatment. ${ }^{157}$

In comparison with normal cells, several receptors, ligands, or extracellular protein motifs, such as integrin $\alpha_{\gamma} \beta_{3}$, transferrin, transmembrane cell surface protein (CD44), and folic acid, are expressed in tumor cells or overexpressed in tumor cells. These substances can serve as specific targets for drug delivery into tumor cells. Fan et al. modified folate on the surface of CDs with $\mathrm{NH}_{2}$ through amide bonds. ${ }^{158} \mathrm{CDs}$ exhibit a high specific targeting capacity for various cancer cells, including OCM-1, MUM2B, and OM431, and this capacity is attributed to the surface folic acid modification of CDs. After CDs are combined with autophagy inhibitors, they are released in the cytoplasm, and a good therapeutic efficiency is achieved in 26 types of tumor cells. In vivo experiments demonstrate that CDs can achieve good therapeutic effect and real-time imaging tumor therapy. The pre-described HA-CDs in Section 3.1 with HA as a carbon source not only act as novel PSs with an $\mathrm{O}_{2}{ }^{--}$generation ability under appropriate light irradiation but also actively target various CD44 overexpressed tumor cell lines because of the specific targeting capability of $\mathrm{HA}$ to CD44. ${ }^{130}$ Therefore, HA-CDs can selectively kill cancer cells with a high CD44 expression.

Intratumoral acidosis is another remarkable character of TME. The lactic acid accumulation caused by the Warburg effect and the slow waste product washout caused by a blocked lymphatic drainage are the main causes of the onset of intratumoral acidosis. ${ }^{159,160}$ Many pH-responsive charge-convertible CD-based drug delivery systems are developed on the basis of intratumoral acidosis. For example, Zhou's group designed low toxicity hybrid PEG-chitosan@CD nanogels, which combine the functional building blocks of fluorescent CDs, thermoresponsive nonlinear PEG, pH-responsive chitosan, and the chemotherapy agent DOX. ${ }^{161}$ Embedded CDs provide hybrid nanogels with a high photothermal ability and a TPF cellular imaging ability to light tumor cells under UV and NIR laser excitation. The protonation of $\mathrm{NH}_{2}$ on chitosan in mild acid and the integrated nonlinear PEG endow hybrid nanogels with $\mathrm{pH}$ and thermal dual-responsive ability to enable the regulated release of the loaded anticancer drug DOX. With hybrid nanogels, the combined synergistic chemo-PTT treatment achieves a high therapeutic efficacy. They also reported another DOX-loaded chitosan-CD hybrid nanogel (CCHN). ${ }^{162}$ As shown in Fig. 16a, CCHNs exhibit efficient NIR photothermal conversion and intelligent drug release in response to NIR light and acidic pH. Zhao et al. prepared CD-Pt(Iv)@PEG(PAH/DMMA) nanoparticles. ${ }^{163}$ The surface modified dimethylmaletic acid (DMMA) of CD-Pt(Iv)@PEG-(PAH/DMMA) hydrolyzes under a mildly acidic condition $(\mathrm{pH}<6.8)$ in a TME to release amino groups, thereby converting the negative surface charge to a positive charge; as such, drug nanocarriers easily 
a

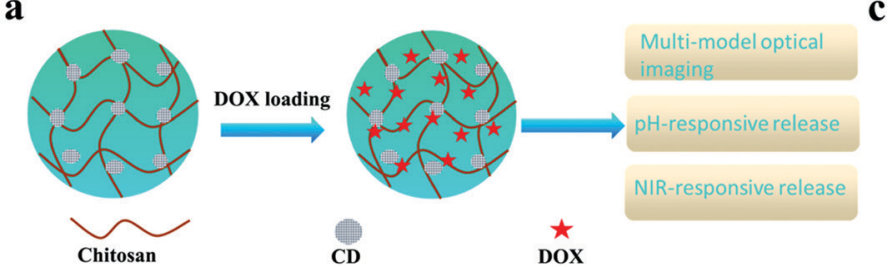

a

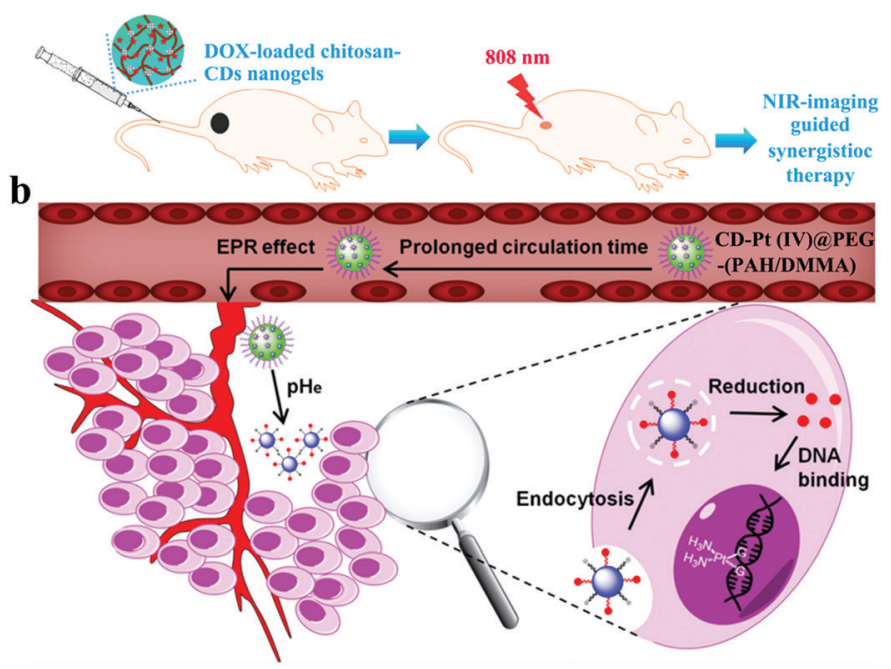

c

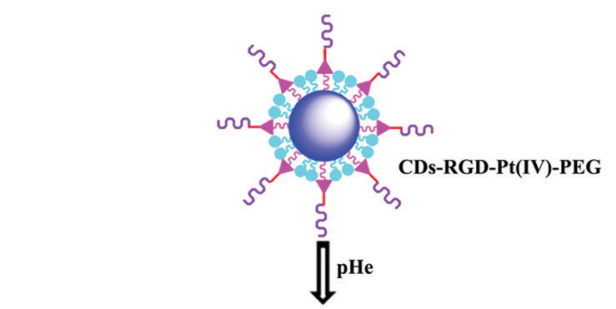

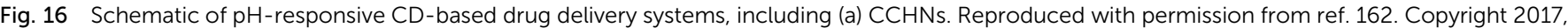

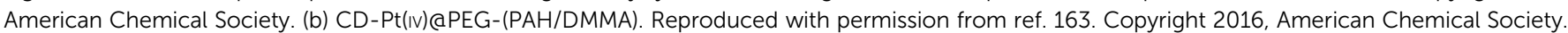
(c) CD-RGD-Pt(Iv)-PEG. Reproduced with permission from ref. 164. Copyright 2016, American Chemical Society.

enter a cancer cell via electrostatic absorption with a negatively charged cell membrane (Fig. 16b). In vitro and in vivo studies demonstrate that drug nanocarriers with a charge conversion property under the stimulation of a TME show an enhanced antitumor efficiency. Zhao's group designed a $\mathrm{pH} /$ redox dualresponsive CD-RGD-Pt(Iv)-PEG nanosystem with fluorescent CDs as imaging-guided drug carriers, cisplatin (Pt, Iv) as a prodrug, and RGD peptide as an active targeting ligand. ${ }^{164}$ Given the hydrolysis of benzoic-imine bond at extracellular tumor $\mathrm{pH}$ (6.5-6.8), the inner targeting RGD peptide of CD-RGD-Pt(Iv)-PEG can be exposed to the effective uptake by cancer cells through the RGD integrin $\alpha \gamma \beta 3$ interaction (Fig. 16c). In vitro results indicate that nanosystems exhibit an enhanced tumor extracellular microenvironment that triggers targeting and anticancer drug delivery. Additionally, $\mathrm{Na}$ and coworkers also prepared pH-sensitive CDs (pSCDs) from citric acid and 1-(3-aminopropyl)imidazole. ${ }^{165}$ Given that imidazole on the surface of CDs can be protonated in an acidic TME (pH 6.5), the surface charge reversion of pSCDs can trigger the effective release of DOX.

Many stimulus-responsive CD-based nanoparticles responsive to highly expressed GSH in a TME have been constructed to facilitate drug release. Xue's group developed a dual-responsive nanoplatform by using a PEGylated shell and a fluorescent CD core with pre-modified DMMA containing disulfide-bondbearing hyper-branched ploy(amido amine; HPAP) as shown in Fig. 17a. ${ }^{166}$ DMMA hydrolysis in a TME causes the surface charge reversal of nanoplatforms to facilitate their accumulation in tumor tissues. Then, GSH can degrade the S-S bond on the surface of CDs, causing the nanoplatform to shrink and promoting the loaded gene drug release in the tumor site. Wang et al. developed a tumor-targeted and multistimulus-responsive CDbased drug delivery system. ${ }^{167}$ As shown in Fig. 17b, the oxidized mesoporous carbon nanoparticles ( $\mathrm{MCN}-\mathrm{COOH})$ with an excellent photothermal property are utilized to encapsulate DOX through the $\mathrm{S}-\mathrm{S}$ bond, and then their outer surfaces are capped with CDS (CDHA) with a tumor targeting ability. The CDHA can prevent premature drug release and enable the visualization of tumor sites and the anticancer drug to actively deliver to tumor cells. The drug release of MC-CDHA/DOX systems can be triggered by pH and GSH in a TME for a highly efficient chemophotothermal synergistic therapy under NIR light irradiation.

Hypoxia caused by the rapid proliferation of cancer cells strongly restricts the efficiency of type II PDT for tumors. CD-based photodynamic nanoplatforms with an $\mathrm{O}_{2}$ generation ability have been developed to solve this problem. The $\mathrm{H}_{2} \mathrm{O}_{2}$ concentration at tumor sites is usually higher than that of normal tissues and can be catalyzed by $\mathrm{MnO}_{2}$ to $\mathrm{O}_{2}$ under acidosis in a TME for enhanced PDT. ${ }^{168,169}$ Recently, our group developed a $\mathrm{CD} / \mathrm{MnO}_{2}$-PEG nanohybrid with previous PT2-CD PS as a reducing agent and potassium permanganate as an oxidation agent. ${ }^{170}$ The as-prepared nanohybrids exhibit an "off" state in a normal physiological environment. However, given the high sensitivity of $\mathrm{MnO}_{2}$ to a TME, CD/MnO $2-\mathrm{PEG}$ nanohybrids change from an "off" state to an "on" state with synchronously enhanced fluorescence, ${ }^{1} \mathrm{O}_{2}$ generation, and $\mathrm{MR}$ imaging signal. The $\mathrm{MnO}_{2}-\mathrm{H}_{2} \mathrm{O}_{2}$ redox reaction at a reduced 
$\mathbf{a}$

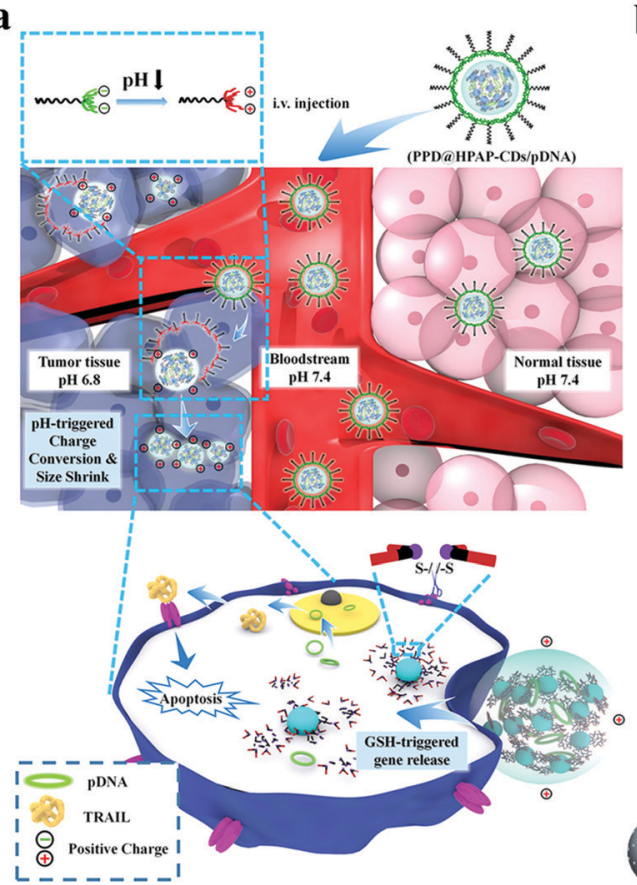

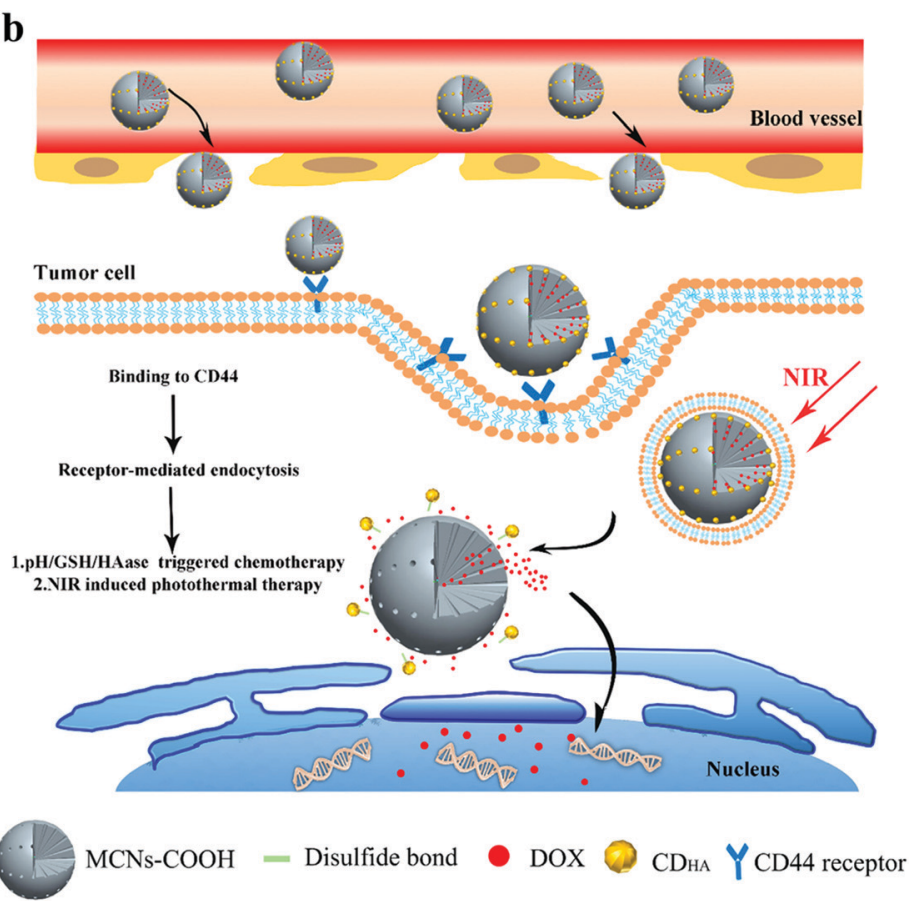

Fig. 17 (a) Schematic of the cascaded response of PPD (aHPAP-CD/pDNA during delivery. Reproduced with permission from ref. 161. Copyright 2018, American Chemical Society. (b) Synthetic schematic of MC-CDHA/DOX as cancer thermo-chemotherapy nanoplatform. Reproduced with permission from ref. 162. Copyright 2019, Elsevier.

$\mathrm{pH}$ in situ generates massive $\mathrm{O}_{2}$ in tumor sites, significantly promoting the PDT efficacy of $\mathrm{CD} / \mathrm{MnO}_{2}$-PEG nanohybrids. Subsequently, we developed versatile MnO-doped CDs as acidic $\mathrm{H}_{2} \mathrm{O}_{2}$-driven oxygenerators to regulate tumor hypoxia for enhanced PDT (Fig. 18). ${ }^{101} \mathrm{Mn}$ can catalyze overexpressed $\mathrm{H}_{2} \mathrm{O}_{2}$ in a tumor region to $\mathrm{O}_{2}$ under a mildly acidic TME. Thus, tumor hypoxia is prevent, and PDT efficacy is synergistically enhanced. After the assembly with DSPE-PEG, Mn-CD assembly exhibits an excellent capability for NIR FL $(745 \mathrm{~nm})$ and $T_{1}$-weighted MR imaging to guide cancer treatment.

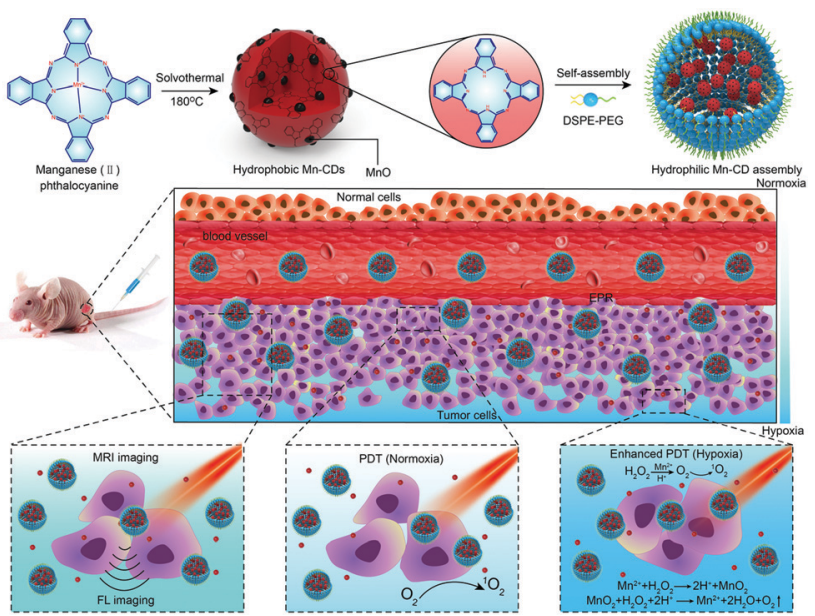

Fig. 18 Schematic of $\mathrm{MnO}$-doped $\mathrm{CDs}$ as an acidic $\mathrm{H}_{2} \mathrm{O}_{2}$-driven oxygenerator for enhancing the anticancer efficiency of PDT. Reproduced with permission from ref. 101. Copyright 2018, Wiley-VCH.
In contrast to oxygen-dependent of PDT II, the type I PDT pathway can occur in a hypoxic environment. This pathway involves electron transfer and radical (e.g. $\mathrm{O}_{2}{ }^{--}$and $\left.{ }^{\bullet} \mathrm{OH}\right)$ formation from direct ionization events. In this case, unlike ${ }^{1} \mathrm{O}_{2}$, which is derived from $\mathrm{O}_{2},{ }^{\bullet} \mathrm{OH}$ is generated from $\mathrm{H}_{2} \mathrm{O}$, thereby minimizing oxygen dependence. In addition, PTT, which converts the energy of electrons to heat, is an important oxygen-independent phototherapy. Therefore, to enhance the therapeutic efficiency in hypoxic TMEs, Zhao's group designed a hybrid nanomedicine (CD-IR820-Aptamer) against hypoxic tumors. ${ }^{171}$ CD-IR820-Aptamer constructed with CDs, IR820 dye, and antivascular endothelial growth factor simultaneously exhibits type I PDT, type II PDT, and PTT under single $808 \mathrm{~nm}$ laser irradiation. Through these three phototherapeutic pathways, the proposed CD-IR820-Aptamer can achieve a high efficiency for the treatment of solid tumors. Additionally, based on natural photosynthesis, a new $\mathrm{O}_{2}$ generation strategy via light-driven water splitting was introduced by Zhang et al. to relieve hypoxia in tumors. ${ }^{172}$ CDs doped with $\mathrm{C}_{3} \mathrm{~N}_{4}$ are prepared with a strong absorption in the red region, and this property is beneficial to light-driven water splitting to produce $\mathrm{O}_{2}$. After a $\mathrm{C}_{3} \mathrm{~N}_{4}$-based nanocomposite (PCCN) is assembled with an amphipathic polymer, consisting of PS protoporphyrin IX and the tumor-targeting sequence RGD with PEG as a linker, PCCN can accumulate in tumor tissues through the active RGD targeting effect and split water to produce $\mathrm{O}_{2}$ and ${ }^{1} \mathrm{O}_{2}$ through the energy transmission of PSs under $630 \mathrm{~nm}$ laser irradiation. This strategy can effectively overcome the restriction of tumor hypoxia in PDT and inhibit solid tumor growth. 


\section{Concluding remarks and perspectives}

Although in-depth studies on CD-based cancer nanotheranostics have gained rapid growth, exploiting smart CDs for cancer theranostics remains in infancy. Current nanotheranostics based on CDs are far from being applied to clinical uses. Therefore, remarkable efforts should be devoted to implementing such promising, low toxic, and good biocompatible carbon nanomaterials for basic biomedical studies and potential clinical uses in the future.

(1) Nanotheranostics should be exploited to develop simple and low-cost approaches for the mass production of CDs. Moreover, some issues, such as size control, batch-to-batch reproducibility, and purification, associated with realizing large-scale synthesis should be addressed.

(2) The new progress toward the development of CDs as multifunctional theranostics and the expansion of CD-related studies in biomedical fields are expected to be further achieved. To this end, the design of new precursors as carbon sources is urgently needed to prepare CDs with novel properties, such as strong emission in NIR-I (700-900 nm) or NIR-II (1100-1600 nm) with a high QY, multimodal bioimaging functions combining at least three of the imaging modalities of FL, PA, MRI, and CT; the new therapeutic modalities (sonodynamic, electrodynamic, and chemodynamic therapies or immunotherapy); and the capability of a TME ( $\mathrm{pH}$, hypoxia, $\mathrm{H}_{2} \mathrm{O}_{2}, \mathrm{GSH}$, and enzyme) response to precisely target and enhance therapeutic effects. In addition to cancer therapy, research on the biomedical use of CDs in other diseases, such as cardiovascular, cerebrovascular, and respiratory system diseases, and wound-healing should be given considerable attention.

(3) Previous reports revealed that CD assemblies can enhance or regulate the properties of an individual CD. For instance, surface properties can be regulated from hydrophobicity to hydrophilicity, and light response in the NIR region can be improved. However, CD assemblies in nanotheranostics have yet to be fully investigated. For example, bioinspired and biomimetic carriers self-assembled by using nanoparticles, peptides, or copolymers as building blocks with features similar to those of biological systems, such as cells, pathogens, and organelles, have been considered more advantageous than conventional nanocarriers. This phenomenon is attributed to their specificity to carry a theranostic moiety to a targeted site, with a high potential to resolve any obstacles encountered in drug delivery. ${ }^{173}$ Constructing bioinspired and biomimetic systems based on CDs via self-assembly is highly expected to resolve the obstacles of current $\mathrm{CD}$ nanotheranostic agents, such as short circulation time in blood, instability in a complex biological environment, and relatively low tumor-homing ability, and selectivity through the EPR effect.

(4) Yan and coworkers first reported that $\mathrm{Fe}_{3} \mathrm{O}_{4}$ NPs possess an intrinsic peroxidase-mimicking activity. ${ }^{174}$ Since then, various nanomaterials, such as noble metal, oxides and carbonaceous nanostrutures have been found to mimic the catalytic activity of different natural enzymes, including peroxidase, oxidase, and catalase, and these namomaterials are called nanozymes. ${ }^{175-178}$ Nanozymes have emerged as novel materials in nanobiomedicine. Studies have also suggested that nanozyme activities can be regulated by $\mathrm{pH}, \mathrm{H}_{2} \mathrm{O}_{2}$, and $\mathrm{GSH}$ concentrations associated with TMEs and by external stimuli such as light, ultrasound, and heat. These collective properties of nanozymes enable them to maximize their diagnostic and therapeutic efficacies against tumors. CDs have been extensively studied as a peroxidase mimic for glucose detection. ${ }^{179}$ However, multistimulus-responsive CDs with enzyme-like activities under physiological conditions for cancer theranostics have yet to be fully explored. Therefore, by combining the catalytic property of nanozyme systems with inherent nature, stimulus-responsive CD nanozymes may show potential for application in tumor nanotheranostics.

(5) Nanotheranostic development focuses on enhancing the efficacy, accuracy, and safety of the diagnosis and treatment of diseases. Therefore, the systematic and long-term studies are still needed to comprehensively explore their biological effects. Moreover, the biodistribution, circulation, translocation, metabolism, biodegradation, and long-term toxicology and secretion of CDs should be investigated in detail. These issues are the most important and challenging ones to translate current CD nanotheranostics from the bench to the bedside.

With the rapid development of CDs, we expect to see more practical uses in various fields not only in nanobiomedicine but also in environment, energy, agriculture, and national security. We believe that these aforementioned unsolved issues will continue to be hopeful and challenging field for further studies on CDs in terms of their basic studies and practical applications.

\section{Conflicts of interest}

There are no conflicts to declare.

\section{Acknowledgements}

This work was supported by the National Natural Science Foundation of China (Grant No. 51902262 and 51972315), the Strategic Priority Research Program of the Chinese Academy of Sciences (Grant No. XDB17000000) and the Fundamental Research Funds for the Central Universities (Grant No. G2018KY0306 and G2019KY05101).

\section{Notes and references}

1 F. Bray, J. Ferlay, I. Soerjomataram, R. L. Siegel, L. A. Torre and A. Jemal, Global cancer statistics 2018: GLOBOCAN estimates of incidence and Mortality Worldwide for 36 Cancers in 185 countries, Ca-Cancer J. Clin., 2018, 68, 394-424.

2 H. Chen, W. Zhang, G. Zhu, J. Xie and X. Chen, Rethinking cancer nanotheranostics, Nat. Rev. Mater., 2017, 2, 17024.

3 Y. Liu, P. Bhattarai, Z. Dai and X. Chen, Photothermal therapy and photoacoustic imaging via nanotheranostics in fighting cancer, Chem. Soc. Rev., 2019, 48, 2053-2108. 
4 G. Nabil, K. Bhise, S. Sau, M. Atef, H. A. El-Banna and A. K. Iyer, Nano-engineered delivery systems for cancer imaging and therapy: recent advances, future direction and patent evaluation, Drug Discovery Today, 2019, 24, 462-491.

5 J. Liu, T. Lecuyer, J. Seguin, N. Mignet, D. Scherman, B. Viana and C. Richard, Imaging and therapeutic applications of persistent luminescence nanomaterials, Adv. Drug Delivery Rev., 2019, 138, 193-210.

6 C. O. Silva, J. O. Pinho, J. M. Lopes, A. J. Almeida, M. M. Gaspar and C. Reis, Current trends in cancer nanotheranostics: metallic, polymeric, and lipid-based systems, Pharmaceutics, 2019, 11, 22.

7 R. K. Singh, K. D. Patel, K. W. Leong and H. W. Kim, Progress in nanotheranostics based on mesoporous silica nanomaterial platforms, ACS Appl. Mater. Interfaces, 2017, 9, 10309-10337.

8 Sonali, M. K. Viswanadh, R. P. Singh, P. Agrawal, A. K. Mehata, D. M. Pawde, Narendra, R. Sonkar and M. S. Muthu, Nanotheranostics: emerging strategies for early diagnosis and therapy of brain cancer, Nanotheranostics, 2018, 2, 70-86.

9 N. Z. Knezevic and G. N. Kaluderovic, Silicon-based nanotheranostics, Nanoscale, 2017, 9, 12821-12829.

10 Y. Chen, Y. Wu, B. Sun, S. Liu and H. Liu, Two-dimensional nanomaterials for cancer nanotheranostics, Small, 2017, 13, 1603446.

11 H. Li, J. Huang, F. Lu, Y. Liu, Y. Song, Y. Sun, J. Zhong, H. Huang, Y. Wang, S. Li, Y. Lifshitz, S.-T. Lee and Z. Kang, Impacts of carbon dots on rice plants: boosting the growth and improving the disease resistance, ACS Appl. Bio Mater., 2018, 1, 663-672.

12 A. Sharma and J. Das, Small molecules derived carbon dots: synthesis and applications in sensing, catalysis, imaging, and biomedicine, J. Nanobiotechnol., 2019, 17, 92.

13 H. Liu, J. Ding, K. Zhang and L. Ding, Construction of biomass carbon dots based fluorescence sensors and their applications in chemical and biological analysis, TrAC, Trends Anal. Chem., 2019, 118, 315-337.

14 G. A. M. Hutton, B. C. M. Martindale and E. Reisner, Carbon dots as photosensitisers for solar-driven catalysis, Chem. Soc. Rev., 2017, 46, 6111-6123.

15 C. Hu, M. Li, J. Qiu and Y. P. Sun, Design and fabrication of carbon dots for energy conversion and storage, Chem. Soc. Rev., 2019, 48, 2315-2337.

16 H. Yu, R. Shi, Y. Zhao, G. I. Waterhouse, L. Z. Wu, C. H. Tung and T. Zhang, Smart utilization of carbon dots in semiconductor photocatalysis, Adv. Mater., 2016, 28, 9454-9477.

17 B. Wang, J. Li, Z. Tang, B. Yang and S. Lu, Near-infrared emissive carbon dots with $33.96 \%$ emission in aqueous solution for cellular sensing and light-emitting diodes, Sci. Bull., 2019, 64, 1285-1292.

18 X. Xu, R. Ray, Y. Gu, H. J. Ploehn, L. Gearheart, K. Raker and W. A. Scrivens, Electrophoretic analysis and purification of fluorescent single-walled carbon nanotube fragments, J. Am. Chem. Soc., 2004, 126, 12736-12737.

19 Y.-P. Sun, B. Zhou, Y. Lin, W. Wang, K. A. S. Fernando, P. Pathak, M. J. Meziani, B. A. Harruff, X. Wang, H. Wang,
P. G. Luo, H. Yang, M. E. Kose, B. Chen, L. M. Veca and S.-Y. Xie, Quantum-sized carbon dots for bright and colorful photoluminescence, J. Am. Chem. Soc., 2006, 128, 7756-7757.

20 W. Meng, X. Bai, B. Wang, Z. Liu, S. Lu and B. Yang, Biomass-derived carbon dots and their applications, Energy Environ. Mater., 2019, 2, 172-192.

21 K.-J. Mintz, Y. Zhou and R.-M. Leblanc, Recent development of carbon quantum dots regarding their optical properties, photoluminescence mechanism, and core structure, Nanoscale, 2019, 11, 4634-4652.

22 S. Zhu, Y. Song, X. Zhao, J. Shao, J. Zhang and B. Yang, The photoluminescence mechanism in carbon dots (graphene quantum dots, carbon nanodots, and polymer dots): current state and future perspective, Nano Res., 2015, 8, 355-381.

23 J. Gao, M. Zhu, H. Huang, Y. Liu and Z. Kang, Advances, challenges and promises of carbon dots, Inorg. Chem. Front., 2017, 4, 1963-1986.

24 C. Xia, S. Zhu, T. Feng, M. Yang and B. Yang, Evolution and synthesis of carbon dots: from carbon dots to carbonized polymer dots, Adv. Sci., 2019, 6, 1901316.

25 S. Lu, L. Sui, J. Liu, S. Zhu, A. Chen, M. Jin and B. Yang, Near-infrared photoluminescent polymer-carbon nanodots with two-photon fluorescence, Adv. Mater., 2017, 29, 1603443.

26 K. Nekoueian, M. Amiri, M. Sillanpaa, F. Marken, R. Boukherroub and S. Szunerits, Carbon-based quantum particles: an electroanalytical and biomedical perspective, Chem. Soc. Rev., 2019, 48, 4281-4316.

27 B. P. Jiang, B. Zhou, Z. Lin, H. Liang and X. C. Shen, Recent advances in carbon nanomaterials for cancer phototherapy, Chem. - Eur. J., 2019, 25, 3993-4004.

28 M. Lan, S. Zhao, W. Li, Z. Lin, L. Zhang, B.-P. Jiang and X.-C. Shen, Recent insights into near-infrared light-responsive carbon dots for bioimaging and cancer phototherapy, Inorg. Chem. Front., 2019, 6, 1116-1128.

29 K. Ghosal and A. Ghosh, Carbon dots: the next generation platform for biomedical applications, Mater. Sci. Eng., C, 2019, 96, 887-903.

30 L. Cheng, C. Wang, L. Feng, K. Yang and Z. Liu, Functional nanomaterials for phototherapies of cancer, Chem. Rev., 2014, 114, 10869-10939.

31 R. Vankayala and K.-C. Hwang, Near-infrared-light-activatable nanomaterial-mediated phototheranostic nanomedicines: an emerging paradigm for cancer treatment, Adv. Mater., 2018, 30, 1706320.

32 S. Anwar, H. Ding, M. Xu, X. Hu, Z. Li, J. Wang, L. Liu, L. Jiang, D. Wang, C. Dong, M. Yan, Q. Wang and H. Bi, Recent advances in synthesis, optical properties, and biomedical applications of carbon dots, ACS Appl. Bio Mater., 2019, 2, 2317-2338.

33 B. Zhou, Z. Guo, Z. Lin, L. Zhang, B.-P. Jiang and X.-C. Shen, Recent insights into near-infrared light-responsive carbon dots for bioimaging and cancer phototherapy, Inorg. Chem. Front., 2019, 6, 1116-1128.

34 X. Shi, H. Meng, Y. Sun, L. Qu, Y. Lin, Z. Li and D. Du, Far-red to near-infrared carbon dots: preparation and applications in biotechnology, Small, 2019, 15, 1901507. 
35 J. Du, N. Xu, J. Fan, W. Sun and X. Peng, Carbon dots for in vivo bioimaging and theranostics, Small, 2019, 15, 1805087.

36 D. Li, D. Wang, X. Zhao, W. Xi, A. Zebibula, N. Alifu, J.-F. Chen and J. Qian, Short-wave infrared emitted/excited fluorescence from carbon dots and preliminary applications in bioimaging, Mater. Chem. Front., 2018, 2, 1343-1350.

37 C. Ding, A. Zhu and Y. Tian, Functional surface engineering of C-dots for fluorescent biosensing and in vivo bioimaging, Acc. Chem. Res., 2014, 47, 20-30.

38 K. Hola, Y. Zhang, Y. Wang, E. P. Giannelis, R. Zboril and A. L. Rogach, Carbon dots-emerging light emitters for bioimaging, cancer therapy and optoelectronics, Nano Today, 2014, 9, 590-603.

39 J. Ge, M. Lan, B. Zhou, W. Liu, L. Guo, H. Wang, Q. Jia, G. Niu, X. Huang, H. Zhou, X. Meng, P. Wang, C. S. Lee, W. Zhang and X. Han, A graphene quantum dot photodynamic therapy agent with high singlet oxygen generation, Nat. Commun., 2014, 5, 4596.

40 L. Guo, J. Ge, W. Liu, G. Niu, Q. Jia, H. Wang and P. Wang, Tunable multicolor carbon dots prepared from welldefined polythiophene derivatives and their emission mechanism, Nanoscale, 2016, 8, 729-734.

$41 \mathrm{~L}$. Li and T. Dong, Photoluminescence tuning in carbon dots: surface passivation or/and functionalization, heteroatom doping, J. Mater. Chem. C, 2018, 6, 7944-7970.

42 B. B. Chen, M. L. Liu, C. M. Li and C. Z. Huang, Fluorescent carbon dots functionalization, Adv. Colloid Interface Sci., 2019, 270, 165-190.

43 H. Liu, Y. Zhang and C. Huang, Development of nitrogen and sulfur-doped carbon dots for cellular imaging, J. Pharm. Anal., 2019, 9, 127-132.

44 S. Sun, L. Zhang, K. Jiang, A. Wu and H. Lin, Toward highefficient red emissive carbon dots: facile preparation, unique properties, and applications as multifunctional theranostic agents, Chem. Mater., 2016, 28, 8659-8668.

45 H. Ding, Y. Ji, J.-S. Wei, Q.-Y. Gao, Z.-Y. Zhou and H.-M. Xiong, Facile synthesis of red-emitting carbon dots from pulp-free lemon juice for bioimaging, J. Mater. Chem. B, 2017, 5, 5272-5277.

46 H. Ding, J. S. Wei, N. Zhong, Q. Y. Gao and H. M. Xiong, Highly efficient red-emitting carbon dots with gram-scale yield for bioimaging, Langmuir, 2017, 33, 12635-12642.

47 H. Jia, Z. Wang, T. Yuan, F. Yuan, X. Li, Y. Li, Z. Tan, L. Fan and S. Yang, Electroluminescent warm white light-emitting diodes based on passivation enabled bright red bandgap emission carbon quantum dots, Adv. Sci., 2019, 6, 1900397.

48 W. Yang, H. Zhang, J. Lai, X. Peng, Y. Hu, W. Gu and L. Ye, Carbon dots with red-shifted photoluminescence by fluorine doping for optical bio-imaging, Carbon, 2018, 128, 78-85.

49 H. Ding, X. Zhou, B. Qin, Z. Zhou and Y. Zhao, Highly fluorescent near-infrared emitting carbon dots derived from lemon juice and its bioimaging application, J. Lumin., 2019, 211, 298-304.

50 F. Arcudi, L. Dordevic and M. Prato, Design, synthesis, and functionalization strategies of tailored carbon nanodots, Acc. Chem. Res., 2019, 52, 2070-2079.
51 E. V. Kundelev, N. V. Tepliakov, M. Y. Leonov, V. G. Maslov, A. V. Baranov, A. V. Fedorov, I. D. Rukhlenko and A. L. Rogach, Amino functionalization of carbon dots leads to red emission enhancement, J. Phys. Chem. Lett., 2019, 10, 5111-5116.

52 P. Namdari, B. Negahdari and A. Eatemadi, Synthesis, properties and biomedical applications of carbon-based quantum dots: an updated review, Biomed. Pharmacother., 2017, 87, 209-222.

53 V. Nguyen, J. Si, L. Yan and X. Hou, Direct demonstration of photoluminescence originated from surface functional groups in carbon nanodots, Carbon, 2016, 108, 268-273.

54 F. Yan, Y. Jiang, X. Sun, Z. Bai, Y. Zhang and X. Zhou, Surface modification and chemical functionalization of carbon dots: a review, Mikrochim. Acta, 2018, 185, 424.

55 X. Miao, D. Qu, D. Yang, B. Nie, Y. Zhao, H. Fan and Z. Sun, Synthesis of carbon dots with multiple color emission by controlled graphitization and surface functionalization, Adv. Mater., 2018, 30, 1704740.

56 D. Li, P. Jing, L. Sun, Y. An, X. Shan, X. Lu, D. Zhou, D. Han, D. Shen, Y. Zhai, S. Qu, R. Zboril and A. L. Rogach, Near-infrared excitation/emission and multiphoton-induced fluorescence of carbon dots, Adv. Mater., 2018, 30, 1705913.

57 W. Ren, S. Chen, S. Li, Y. Zhang, J. Liu, M. Guan, H. Yang, N. Li, C. Han, T. Li, Z. Zhao and J. Ge, Photoluminescence enhancement of carbon dots by surfactants at room temperature, Chem. - Eur. J., 2018, 24, 15806-15811.

58 Y. Zhang, P. Zhuo, H. Yin, Y. Fan, J. Zhang, X. Liu and Z. Chen, Solid-state fluorescent carbon dots with aggregation-induced yellow emission for white light-emitting diodes with high luminous efficiencies, ACS Appl. Mater. Interfaces, 2019, 11, 24395-24403.

59 Z. X. Liu, Z. L. Wu, M. X. Gao, H. Liu and C. Z. Huang, Carbon dots with aggregation induced emission enhancement for visual permittivity detection, Chem. Commun., 2016, 52, 2063-2066.

60 Q. Wang, S. Zhang, B. Wang, X. Yang, B. Zou, B. Yang and S. Lu, Pressure-triggered aggregation-induced emission enhancement in red emissive amorphous carbon dots, Nanoscale Horiz., 2019, 4, 1227-1231.

61 H. Yang, Y. Liu, Z. Guo, B. Lei, J. Zhuang, X. Zhang, Z. Liu and $\mathrm{C}$. $\mathrm{Hu}$, Hydrophobic carbon dots with blue dispersed emission and red aggregation-induced emission, Nat. Commun., 2019, 10, 1789.

62 S. Zhu, R. Tian, A. L. Antaris, X. Chen and H. Dai, Nearinfrared-II molecular dyes for cancer imaging and surgery, Adv. Mater., 2019, 31, 1900321.

$63 \mathrm{~J} . \mathrm{Li}$ and $\mathrm{K} . \mathrm{Pu}$, Development of organic semiconducting materials for deep-tissue optical imaging, phototherapy and photoactivation, Chem. Soc. Rev., 2019, 48, 38-71.

64 A. L. Antaris, H. Chen, K. Cheng, Y. Sun, G. Hong, C. Qu, S. Diao, Z. Deng, X. Hu, B. Zhang, X. Zhang, O. K. Yaghi, Z. R. Alamparambil, X. Hong, Z. Cheng and H. Dai, A smallmolecule dye for NIR-II imaging, Nat. Mater., 2015, 15, 235-242.

65 J. Zhao, D. Zhong and S. Zhou, NIR-I-to-NIR-II fluorescent nanomaterials for biomedical imaging and cancer therapy, J. Mater. Chem. B, 2018, 6, 349-365. 
66 T. Yang, Y. Tang, L. Liu, X. Lv, Q. Wang, H. Ke, Y. Deng, H. Yang, X. Yang, G. Liu, Y. Zhao and H. Chen, Sizedependent $\mathrm{Ag}_{2} \mathrm{~S}$ nanodots for second near-infrared fluorescence/photoacoustics imaging and simultaneous photothermal therapy, ACS Nano, 2017, 11, 1848-1857.

67 J. Xu, A. Gulzar, P. Yang, H. Bi, D. Yang, S. Gai, F. He, J. Lin, B. Xing and D. Jin, Recent advances in near-infrared emitting lanthanide-doped nanoconstructs: mechanism, design and application for bioimaging, Coord. Chem. Rev., 2019, 381, 104-134.

68 H. Liu, G. Hong, Z. Luo, J. Chen, J. Chang, M. Gong, H. He, J. Yang, X. Yuan, L. Li, X. Mu, J. Wang, W. Mi, J. Luo, J. Xie and X. D. Zhang, Atomic-precision gold clusters for NIR-II imaging, Adv. Mater., 2019, 31, 1901015.

69 Y. Li, G. Bai, S. Zeng and J. Hao, Theranostic carbon dots with innovative NIR-II emission for in vivo renal-excreted optical imaging and photothermal therapy, ACS Appl. Mater. Interfaces, 2019, 11, 4737-4744.

70 H. Wang, Q. Mu, K. Wang, R. A. Revia, C. Yen, X. Gu, B. Tian, J. Liu and M. Zhang, Nitrogen and boron dualdoped graphene quantum dots for near-infrared second window imaging and photothermal therapy, Appl. Mater. Today, 2019, 14, 108-117.

71 Y. Chen, R. Guan, C. Zhang, J. Huang, L. Ji and H. Chao, Twophoton luminescent metal complexes for bioimaging and cancer Phototherapy, Coord. Chem. Rev., 2016, 310, 16-40.

72 C.-L. Sun, J. Li, X.-Z. Wang, R. Shen, S. Liu, J.-Q. Jiang, T. Li, Q.-W. Song, Q. Liao, H.-B. Fu, J.-N. Yao and H.-L. Zhang, Rational design of organic probes for turn-on two-photon excited fluorescence imaging and photodynamic therapy, Chem, 2019, 5, 600-616.

73 X. Lou, Z. Zhao and B. Z. Tang, Organic dots based on AIEgens for two-photon fluorescence bioimaging, Small, 2016, 12, 6430-6450.

74 Y. Ni, H. Zhang, C. Chai, B. Peng, A. Zhao, J. Zhang, L. Li, C. Zhang, B. Ma, H. Bai, K. L. Lim and W. Huang, Mitochondria-targeted two-photon fluorescent photosensitizers for cancer cell apoptosis via spatial selectability, Adv. Healthcare Mater., 2019, 8, 1900212.

75 L. Cao, X. Wang, M. J. Meziani, F. Lu, H. Wang, P. G. Luo, Y. Lin, B. A. Harruff, L. M. Veca, D. Murray, S.-Y. Xie and Y.-P. Sun, Carbon dots for multiphoton bioimaging, J. Am. Chem. Soc., 2007, 129, 11318-11319.

76 S. Zhu, J. Zhang, S. Tang, C. Qiao, L. Wang, H. Wang, X. Liu, B. Li, Y. Li, W. Yu, X. Wang, H. Sun and B. Yang, Surface chemistry routes to modulate the photoluminescence of graphene quantum dots: from fluorescence mechanism to up-conversion bioimaging applications, Adv. Funct. Mater., 2012, 22, 4732-4740.

77 Q. Liu, B. Guo, Z. Rao, B. Zhang and J. R. Gong, Strong twophoton-induced fluorescence from photostable, biocompatible nitrogen-doped graphene quantum dots for cellular and deep-tissue imaging, Nano Lett., 2013, 13, 2436-2441.

78 L. Pan, S. Sun, L. Zhang, K. Jiang and H. Lin, Near-infrared emissive carbon dots for two-photon fluorescence bioimaging, Nanoscale, 2016, 8, 17350-17356.
79 M. Lan, S. Zhao, Z. Zhang, L. Yan, L. Guo, G. Niu, J. Zhang, J. Zhao, H. Zhang, P. Wang, G. Zhu, C.-S. Lee and W. Zhang, Two-photon-excited near-infrared emissive carbon dots as multifunctional agents for fluorescence imaging and photothermal therapy, Nano Res., 2017, 10, 3113-3123.

80 B. R. Smith and S. S. Gambhir, Nanomaterials for in vivo imaging, Chem. Rev., 2017, 117, 901-986.

$81 \mathrm{~L}$. Bu, B. Shen and Z. Cheng, Fluorescent imaging of cancerous tissues for targeted surgery, Adv. Drug Delivery Rev., 2014, 76, 21-38.

82 J. Weber, P. C. Beard and S. E. Bohndiek, Contrast agents for molecular photoacoustic imaging, Nat. Methods, 2016, 13, 639-650.

83 U. Chitgupi, N. Nyayapathi, J. Kim, D. Wang, B. Sun, C. Li, K. Carter, W. C. Huang, C. Kim, J. Xia and J. F. Lovell, Surfactant-stripped micelles for NIR-II photoacoustic imaging through $12 \mathrm{~cm}$ of breast tissue and whole human breasts, $A d v$. Mater., 2019, 31, 1902279.

84 S. Wang, J. Lin, T. Wang, X. Chen and P. Huang, Recent advances in photoacoustic imaging for deep-tissue biomedical applications, Theranostics, 2016, 6, 2394-2413.

85 R. Zhang, Y. Xu, Y. Zhang, H. S. Kim, A. Sharma, J. Gao, G. Yang, J. S. Kim and Y. Sun, Rational design of a multifunctional molecular dye for dual-modal NIR-II/photoacoustic imaging and photothermal therapy, Chem. Sci., 2019, 10, 8348-8353.

86 W. Hou, J. W. H. Lou, J. Bu, E. Chang, L. Ding, M. Valic, H. H. Jeon, D. M. Charron, C. Coolens, D. Cui, J. Chen and G. Zheng, A nanoemulsion with a porphyrin shell for cancer theranostics, Angew. Chem., Int. Ed., 2019, 58, 14974-14978.

87 W. Wang, T. Jing, X. Xia, L. Tang, Z. Huang, F. Liu, Z. Wang, H. Ran, M. Li and J. Xia, Melanin-loaded biocompatible photosensitive nanoparticles for controlled drug release in combined photothermal-chemotherapy guided by photoacoustic/ultrasound dual-modality imaging, Biomater. Sci., 2019, 7, 4060-4074.

$88 \mathrm{~J}$. Li, J. Rao and K. Pu, Recent progress on semiconducting polymer nanoparticles for molecular imaging and cancer phototherapy, Biomaterials, 2018, 155, 217-235.

89 J. Ge, Q. Jia, W. Liu, L. Guo, Q. Liu, M. Lan, H. Zhang, $\mathrm{X}$. Meng and P. Wang, Red-emissive carbon dots for fluorescent, photoacoustic, and thermal theranostics in living mice, Adv. Mater., 2015, 27, 4169-4177.

90 C. Lee, W. Kwon, S. Beack, D. Lee, Y. Park, H. Kim, S. K. Hahn, S. W. Rhee and C. Kim, Biodegradable nitrogen-doped carbon nanodots for non-invasive photoacoustic imaging and photothermal therapy, Theranostics, 2016, 6, 2196-2208.

91 Q. Jia, X. Zheng, J. Ge, W. Liu, H. Ren, S. Chen, Y. Wen, H. Zhang, J. Wu and P. Wang, Synthesis of carbon dots from Hypocrella bambusae for bimodal fluorescence/ photoacoustic imaging-guided synergistic photodynamic/ photothermal therapy of cancer, J. Colloid Interface Sci., 2018, 526, 302-311.

92 G. Xu, X. Bao, J. Chen, B. Zhang, D. Li, D. Zhou, X. Wang, C. Liu, Y. Wang and S. Qu, In vivo tumor photoacoustic 
imaging and photothermal therapy based on supra-(carbon nanodots), Adv. Healthcare Mater., 2019, 8, 1800995.

93 F. Wu, H. Su, Y. Cai, W.-K. Wong, W. Jiang and X. Zhu, Porphyrin-implanted carbon nanodots for photoacoustic imaging and in vivo breast cancer ablation, ACS Appl. Bio Mater., 2018, 1, 110-117.

94 H. Chen, Y. Qiu, D. Ding, H. Lin, W. Sun, G. D. Wang, W. Huang, W. Zhang, D. Lee, G. Liu, J. Xie and X. Chen, Construction of iron oxide nanoparticle-based hybrid platforms for tumor imaging and therapy, Adv. Mater., 2018, 30, 1802748.

95 Y. Hu, S. Mignani, J. P. Majoral, M. Shen and X. Shi, Nanoscale metal-organic frameworks for therapeutic, imaging, and sensing applications, Chem. Soc. Rev., 2018, 47, 1874-1900.

96 K. Lu, T. Aung, N. Guo, R. Weichselbaum and W. Lin, Gadolinium-encapsulated graphene carbon nanotheranostics for imaging-guided photodynamic therapy, Adv. Mater., 2018, 30, 1707634.

97 H. Chen, G. D. Wang, W. Tang, T. Todd, Z. Zhen, C. Tsang, K. Hekmatyar, T. Cowger, R. Hubbard, W. Zhang, J. Stickney, B. Shen and J. Xie, Gd-encapsulated carbonaceous dots with efficient renal clearance for magnetic resonance imaging, Adv. Mater., 2014, 26, 6761-6766.

98 S. A. Rub Pakkath, S. S. Chetty, P. Selvarasu, A. Vadivel Murugan, Y. Kumar, L. Periyasamy, M. Santhakumar, S. R. Sadras and K. Santhakumar, Transition metal ion $\left(\mathrm{Mn}^{2+}\right.$, $\mathrm{Fe}^{2+}, \mathrm{Co}^{2+}$, and $\mathrm{Ni}^{2+}$ )-doped carbon dots synthesized via microwave-assisted pyrolysis: a potential nanoprobe for magneto-fluorescent dual-modality bioimaging, ACS Biomater. Sci. Eng., 2018, 4, 2582-2596.

99 D. Bouzas-Ramos, J. Cigales Canga, J. C. Mayo, R. M. Sainz, J. Ruiz Encinar and J. M. Costa-Fernandez, Carbon quantum dots codoped with nitrogen and lanthanides for multimodal imaging, Adv. Funct. Mater., 2019, 29, 1903884.

100 J. Sui, G. Liu, Y. Song, D. Li, X. Dong, J. Wang and W. Yu, Integrating photoluminescence, magnetism and thermal conversion for potential photothermal therapy and dual-modal bioimaging, J. Colloid Interface Sci., 2018, 510, 292-301.

101 Q. Jia, J. Ge, W. Liu, X. Zheng, S. Chen, Y. Wen, H. Zhang and P. Wang, A magnetofluorescent carbon dot assembly as an acidic $\mathrm{H}_{2} \mathrm{O}_{2}$-driven oxygenerator to regulate tumor hypoxia for simultaneous bimodal imaging and enhanced photodynamic therapy, Adv. Mater., 2018, 30, 1706090.

102 N. Nimi, A. Saraswathy, S. S. Nazeer, N. Francis, S. J. Shenoy and R. S. Jayasree, Multifunctional hybrid nanoconstruct of zerovalent iron and carbon dots for magnetic resonance angiography and optical imaging: an in vivo study, Biomaterials, 2018, 171, 46-56.

103 J. Wang, X. Hu, H. Ding, X. Huang, M. Xu, Z. Li, D. Wang, X. Yan, Y. Lu, Y. Xu, Y. Chen, P. C. Morais, Y. Tian, R. Q. Zhang and $\mathrm{H}$. Bi, Fluorine and nitrogen co-doped carbon dot complexation with $\mathrm{Fe}(\mathrm{III})$ as a $T_{1}$ contrast agent for magnetic resonance imaging, ACS Appl. Mater. Interfaces, 2019, 11, 18203-18212.

104 H. Wang, R. Revia, K. Wang, R. J. Kant, Q. Mu, Z. Gai, K. Hong and M. Zhang, Paramagnetic properties of metal-free boron-doped graphene quantum dots and their application for safe magnetic resonance imaging, Adv. Mater., 2017, 29, 1605416.

105 J. Zhang, Y. Yuan, M. Gao, Z. Han, C. Chu, Y. Li, P. C. M. van Zijl, M. Ying, J. W. M. Bulte and G. Liu, Carbon dots as a new class of diamagnetic chemical exchange saturation transfer (diaCEST) MRI contrast agents, Angew. Chem., Int. Ed., 2019, 58, 9871-9875.

106 X. Miao, X. Yan, D. Qu, D. Li, F. F. Tao and Z. Sun, Red emissive sulfur, nitrogen codoped carbon dots and their application in ion detection and theraonostics, ACS Appl. Mater. Interfaces, 2017, 9, 18549-18556.

107 Y. Su, S. Lu, P. Gao, M. Zheng and Z. Xie, BODIPY@carbon dot nanocomposites for enhanced photodynamic activity, Mater. Chem. Front., 2019, 3, 1747-1753.

108 P. Devi, S. Saini and K. H. Kim, The advanced role of carbon quantum dots in nanomedical applications, Biosens. Bioelectron., 2019, 141, 111158.

109 P. Huang, J. Lin, X. Wang, Z. Wang, C. Zhang, M. He, K. Wang, F. Chen, Z. Li, G. Shen, D. Cui and X. Chen, Light-triggered theranostics based on photosensitizer conjugated carbon dots for simultaneous enhanced fluorescence imaging and photodynamic therapy, Adv. Mater., 2012, 24, 5104-5110.

110 J. Tang, B. Kong, H. Wu, M. Xu, Y. Wang, Y. Wang, D. Zhao and G. Zheng, Carbon nanodots featuring efficient FRET for real-time monitoring of drug delivery and two-photon imaging, Adv. Mater., 2013, 25, 6569-6574.

111 M. Zheng, S. Liu, J. Li, D. Qu, H. Zhao, X. Guan, X. Hu, Z. Xie, X. Jing and Z. Sun, Integrating oxaliplatin with highly luminescent carbon dots: an unprecedented theranostic agent for personalized medicine, Adv. Mater., 2014, 26, 3554-3560.

112 S. Monro, K. L. Colon, H. Yin, J. Roque, P. Konda, S. Gujar, R. P. Thummel, L. Lilge, C. G. Cameron and S. A. McFarland, Transition metal complexes and photodynamic therapy from a tumor-centered approach: challenges, opportunities, and highlights from the development of TLD1433, Chem. Rev., 2019, 119, 797-828.

113 Y. Liu, X. Meng and W. Bu, Upconversion-based photodynamic cancer therapy, Coord. Chem. Rev., 2019, 379, 82-98.

114 W. H. Chen, G. F. Luo and X. Z. Zhang, Recent advances in subcellular targeted cancer therapy based on functional materials, Adv. Mater., 2019, 31, 1802725.

115 Q. Li, Y. Li, T. Min, J. Gong, L. Du, D. L. Phillips, J. Liu, J. W. Y. Lam, H. H. Y. Sung, I. D. Williams, R. T. K. Kwok, C. L. Ho, K. Li, J. Wang and B. Z. Tang, Time-dependent photodynamic therapy for multiple targets: a highly efficient AIE-active photosensitizer for selective bacterial elimination and cancer cell ablation, Angew. Chem., Int. Ed., 2019, 58, 2-10.

116 J. Karges, U. Basu, O. Blacque, H. Chao and G. Gasser, Polymeric encapsulation of novel homoleptic bis(dipyrrinato) Zinc(II) complexes with long lifetimes for applications as photodynamic therapy photosensitisers, Angew. Chem., Int. Ed., 2019, 58, 14334-14340. 
117 X. Li, S. Lee and J. Yoon, Supramolecular photosensitizers rejuvenate photodynamic therapy, Chem. Soc. Rev., 2018, 47, 1174-1188.

118 I. L. Christensen, Y. P. Sun and P. Juzenas, Carbon dots as antioxidants and prooxidants, J. Biomed. Nanotechnol., 2011, 7, 667-676.

119 Z. M. Markovic, B. Z. Ristic, K. M. Arsikin, D. G. Klisic, L. M. Harhaji-Trajkovic, B. M. Todorovic-Markovic, D. P. Kepic, T. K. Kravic-Stevovic, S. P. Jovanovic, M. M. Milenkovic, D. D. Milivojevic, V. Z. Bumbasirevic, M. D. Dramicanin and V. S. Trajkovic, Graphene quantum dots as autophagyinducing photodynamic agents, Biomaterials, 2012, 33, 7084-7092.

120 M. Guan, J. Li, Q. Jia, J. Ge, D. Chen, Y. Zhou, P. Wang, T. Zou, M. Zhen, C. Wang and C. Shu, A versatile and clearable nanocarbon theranostic based on carbon dots and gadolinium metallofullerene nanocrystals, $A d v$. Healthcare Mater., 2016, 5, 2283-2294.

121 Q. Jia, J. Ge, W. Liu, L. Guo, X. Zheng, S. Chen, M. Chen, S. Liu, L. Zhang, M. Wang, H. Zhang and P. Wang, Selfassembled carbon dot nanosphere: a robust, near-infrared light-responsive, and vein injectable photosensitizer, $A d v$. Healthcare Mater., 2017, 6, 1601419.

122 F. Wu, J. Chen, Z. Li, H. Su, K. C.-F. Leung, H. Wang and X. Zhu, Red/near-infrared emissive metalloporphyrinbased nanodots for magnetic resonance imaging-guided photodynamic therapy in vivo, Part. Part. Syst. Charact., 2018, 35, 1800208.

123 X. T. Zheng, Y. C. Lai and Y. N. Tan, Nucleotide-derived theranostic nanodots with intrinsic fluorescence and singlet oxygen generation for bioimaging and photodynamic therapy, Nanoscale Adv., 2019, 1, 2250-2257.

124 Y. Li, X. Zheng, X. Zhang, S. Liu, Q. Pei, M. Zheng and Z. Xie, Porphyrin-based carbon dots for photodynamic therapy of hepatoma, Adv. Healthcare Mater., 2017, 6, 1600924.

125 H. He, X. Zheng, S. Liu, M. Zheng, Z. Xie, Y. Wang, M. Yu and X. Shuai, Diketopyrrolopyrrole-based carbon dots for photodynamic therapy, Nanoscale, 2018, 10, 10991-10998.

126 Y. Wen, Q. Jia, F. Nan, X. Zheng, W. Liu, J. Wu, H. Ren, J. Ge and P. Wang, Pheophytin derived near-infrared-light responsive carbon dot assembly as a new phototheranotic agent for bioimaging and photodynamic therapy, Chem. Asian J., 2019, 14, 2162-2168.

127 N. Kaur, V. Sharma, P. Tiwari, A. K. Saini and S. M. Mobin, "Vigna radiate" based green C-dots: photo-triggered theranostics, fluorescent sensor for extracellular and intracellular iron(III) and multicolor live cell imaging probe, Sens. Actuators, B, 2019, 291, 275-286.

128 X. Li, N. Kwon, T. Guo, Z. Liu and J. Yoon, Innovative strategies for hypoxic-tumor photodynamic therapy, Angew. Chem., Int. Ed., 2018, 57, 11522-11531.

129 Y. Zhang, C. Yang, D. Yang, Z. Shao, Y. Hu, J. Chen, L. Yuwen, L. Weng, Z. Luo and L. Wang, Reduction of graphene oxide quantum dots to enhance the yield of reactive oxygen species for photodynamic therapy, Phys. Chem. Chem. Phys., 2018, 20, 17262-17267.
130 L. Zhang, Z. Lin, Y. X. Yu, B. P. Jiang and X. C. Shen, Multifunctional hyaluronic acid-derived carbon dots for self-targeted imaging-guided photodynamic therapy, J. Mater. Chem. B, 2018, 6, 6534-6543.

131 J. Chen, C. Ning, Z. Zhou, P. Yu, Y. Zhu, G. Tan and C. Mao, Nanomaterials as photothermal therapeutic agents, Prog. Mater. Sci., 2019, 99, 1-26.

132 J. R. Choi, K. W. Yong, J. Y. Choi, A. Nilghaz, Y. Lin, J. Xu and $\mathrm{X}$. Lu, Black phosphorus and its biomedical applications, Theranostics, 2018, 8, 1005-1026.

133 H. S. Jung, P. Verwilst, A. Sharma, J. Shin, J. L. Sessler and J. S. Kim, Organic molecule-based photothermal agents: an expanding photothermal therapy universe, Chem. Soc. Rev., 2018, 47, 2280-2297.

134 H. Zhu, P. Cheng, P. Chen and K. Pu, Recent progress in the development of nearinfrared organic photothermal and photodynamic nanotherapeutics, Biomater. Sci., 2018, 6, 746-765.

135 X. Bao, Y. Yuan, J. Chen, B. Zhang, D. Li, D. Zhou, P. Jing, G. Xu, Y. Wang, K. Hola, D. Shen, C. Wu, L. Song, C. Liu, R. Zboril and S. Qu, In vivo theranostics with nearinfraredemitting carbon dots-highly efficient photothermal therapy based on passive targeting after intravenous administration, Light: Sci. Appl., 2018, 7, 91.

136 B. Geng, D. Yang, D. Pan, L. Wang, F. Zheng, W. Shen, C. Zhang and X. Li, NIR-responsive carbon dots for efficient photothermal cancer therapy at low power densities, Carbon, 2018, 134, 153-162.

137 M. Zheng, Y. Li, S. Liu, W. Wang, Z. Xie and X. Jing, Onepot to synthesize multifunctional carbon dots for near infrared fluorescence imaging and photothermal cancer therapy, ACS Appl. Mater. Interfaces, 2016, 8, 23533-23541.

138 M. Qian, Y. Du, S. Wang, C. Li, H. Jiang, W. Shi, J. Chen, Y. Wang, E. Wagner and R. Huang, Highly Crystalline multicolor carbon nanodots for dual-modal imagingguided photothermal therapy of glioma, ACS Appl. Mater. Interfaces, 2018, 10, 4031-4040.

139 C. Yang, Y. Li, Y. Yang, R. Tong, L. He, E. Long, L. Liang and L. Cai, Multidimensional theranostics for tumor fluorescence imaging, photoacoustic imaging and photothermal treatment based on manganese doped carbon dots, J. Biomed. Nanotechnol., 2018, 14, 1590-1600.

140 J. N. Liu, W. Bu and J. Shi, Chemical design and synthesis of functionalized probes for imaging and treating tumor hypoxia, Chem. Rev., 2017, 117, 6160-6224.

141 J. Xie, L. Gong, S. Zhu, Y. Yong, Z. Gu and Y. Zhao, Emerging strategies of nanomaterial-mediated tumor radiosensitization, Adv. Mater., 2019, 31, 1802244.

142 Y. Dai, C. Xu, X. Sun and X. Chen, Nanoparticle design strategies for enhanced anticancer therapy by exploiting the tumour microenvironment, Chem. Soc. Rev., 2017, 46, 3830-3852.

143 W. Fan, P. Huang and X. Chen, Overcoming the Achilles' heel of photodynamic therapy, Chem. Soc. Rev., 2016, 45, 6488-6519.

144 C. Wang, W. Fan, Z. Zhang, Y. Wen, L. Xiong and X. Chen, Advanced nanotechnology leading the way to multimodal 
imaging-guided precision surgical therapy, Adv. Mater., 2019, 31, 1904329.

145 W. Sang, Z. Zhang, Y. Dai and X. Chen, Recent advances in nanomaterial-based synergistic combination cancer immunotherapy, Chem. Soc. Rev., 2019, 48, 3771-3810.

146 S. Gai, G. Yang, P. Yang, F. He, J. Lin, D. Jin and B. Xing, Recent advances in functional nanomaterials for lighttriggered cancer therapy, Nano Today, 2018, 19, 146-187.

147 Q. Jia, J. Ge, W. Liu, S. Liu, G. Niu, L. Guo, H. Zhang and P. Wang, Gold nanorod@silica-carbon dots as multifunctional phototheranostics for fluorescence and photoacoustic imagingguided synergistic photodynamic/photothermal therapy, Nanoscale, 2016, 8, 13067-13077.

148 S. Sun, J. Chen, K. Jiang, Z. Tang, Y. Wang, Z. Li, C. Liu, A. $\mathrm{Wu}$ and $\mathrm{H}$. Lin, Ce6-modified carbon dots for multimodal-imaging-guided and single-NIR-laser-triggered photothermal/photodynamic synergistic cancer therapy by reduced irradiation power, ACS Appl. Mater. Interfaces, 2019, 11, 5791-5803.

149 C. Scialabba, A. Sciortino, F. Messina, G. Buscarino, M. Cannas, G. Roscigno, G. Condorelli, G. Cavallaro, G. Giammona and N. Mauro, Highly homogeneous biotinylated carbon nanodots: red-emitting nanoheaters as theranostic agents toward precision cancer medicine, ACS Appl. Mater. Interfaces, 2019, 11, 19854-19866.

150 J. Ge, Q. Jia, W. Liu, M. Lan, B. Zhou, L. Guo, H. Zhou, H. Zhang, Y. Wang, Y. Gu, X. Meng and P. Wang, Carbon dots with intrinsic theranostic properties for bioimaging, red-light-triggered photodynamic/photothermal simultaneous therapy in vitro and in vivo, Adv. Healthcare Mater., 2016, 5, 665-675.

151 X.-L. Guo, Z.-Y. Ding, S.-M. Deng, C.-C. Wen, X.-C. Shen, B.-P. Jiang and H. Liang, A novel strategy of transitionmetal doping to engineer absorption of carbon dots for near-infrared photothermal/photodynamic therapies, Carbon, 2018, 134, 519-530.

152 C. Belli, D. Trapani, G. Viale, P. D’Amico, B. A. Duso, P. Della Vigna, F. Orsi and G. Curigliano, A novel strategy of transition-metal doping to engineer absorption of carbon dots for near-infrared photothermal/photodynamic therapies, Cancer Treat. Rev., 2018, 65, 22-32.

153 Z. Tang, Y. Liu, M. He and W. Bu, Chemodynamic therapy: tumour microenvironment mediated Fenton and Fentonlike reactions, Angew. Chem., Int. Ed., 2019, 58, 946-956.

154 J. He, C. Li, L. Ding, Y. Huang, X. Yin, J. Zhang, J. Zhang, C. Yao, M. Liang, R. P. Pirraco, J. Chen, Q. Lu, R. Baldridge, Y. Zhang, M. Wu, R. L. Reis and Y. Wang, Tumor targeting strategies of smart fluorescent nanoparticles and their applications in cancer diagnosis and treatment, $A d v$. Mater., 2019, 31, 1902409.

155 Z. Gu, S. Zhu, L. Yan, F. Zhao and Y. Zhao, Graphene-based smart platforms for combined cancer therapy, Adv. Mater., 2019, 31, 1800662.

156 C. Chu, H. Lin, H. Liu, X. Wang, J. Wang, P. Zhang, H. Gao, C. Huang, Y. Zeng, Y. Tan, G. Liu and X. Chen, Tumor microenvironment-triggered supramolecular system as an in situ nanotheranostic generator for cancer phototherapy, Adv. Mater., 2017, 29, 1605928.

157 H. S. El-Sawy, A. M. Al-Abd, T. A. Ahmed, K. M. El-Say and V. P. Torchilin, Stimuli-responsive nano-architecture drugdelivery systems to solid tumor micromilieu: past, present, and future perspectives, ACS Nano, 2018, 12, 10636-10664.

158 J. Li, S. Yang, Y. Deng, P. Chai, Y. Yang, X. He, X. Xie, Z. Kang, G. Ding, H. Zhou and X. Fan, Emancipating targetfunctionalized carbon dots from autophagy vesicles for a novel visualized tumor therapy, Adv. Funct. Mater., 2018, 28, 1800881.

159 M. R. Horsman and P. Vaupel, Pathophysiological basis for the formation of the tumor microenvironment, Front. Oncol., 2016, 6, 66.

160 M. G. Vander Heiden, L. C. Cantley and C. B. Thompson, Understanding the Warburg effect: the metabolic requirements of cell proliferation, Science, 2009, 324, 1029-1033.

161 H. Wang, J. Di, Y. Sun, J. Fu, Z. Wei, H. Matsui, A. del, C. Alonso and S. Zhou, Biocompatible PEG-chitosan@carbon dots hybrid nanogels for two-photon fluorescence imaging, near-infrared light/pH dual-responsive drug carrier, and synergistic therapy, Adv. Funct. Mater., 2015, 25, 5537-5547.

162 H. Wang, S. Mukherjee, J. Yi, P. Banerjee, Q. Chen and S. Zhou, Biocompatible chitosan-carbon dot hybrid nanogels for NIR imaging-guided synergistic photothermal-chemo therapy, ACS Appl. Mater. Interfaces, 2017, 9, 18639-18649.

163 T. Feng, X. Ai, G. An, P. Yang and Y. Zhao, Charge-convertible carbon dots for imaging guided drug delivery with enhanced in vivo cancer therapeutic efficiency, ACS Nano, 2016, 10, 4410-4420.

164 T. Feng, X. Ai, H. Ong and Y. Zhao, Dual-responsive carbon dots for tumor extracellular microenvironment triggered targeting and enhanced anticancer drug delivery, ACS Appl. Mater. Interfaces, 2016, 8, 18732-18740.

165 J. Seo, J. Lee, C. B. Lee, S. K. Bae and K. Na, Non-polymeric $\mathrm{pH}$-sensitive carbon dots for treatment of tumor, Bioconjugate Chem., 2019, 30, 621-632.

166 H. Zhao, J. Duan, Y. Xiao, G. Tang, C. Wu, Y. Zhang, Z. Liu and W. Xue, Microenvironment-driven cascaded responsive hybrid carbon dots as a multifunctional theranostic nanoplatform for imaging traceable gene precise delivery, Chem. Mater., 2018, 30, 3438-3453.

167 X. Wang, X. Li, Y. Mao, D. Wang, Q. Zhao and S. Wang, Multi-stimuli responsive nanosystem modified by tumortargeted carbon dots for chemophototherapy synergistic therapy, J. Colloid Interface Sci., 2019, 552, 639-650.

168 Q. Chen, L. Feng, J. Liu, W. Zhu, Z. Dong, Y. Wu and Z. Liu, Intelligent albumin- $\mathrm{MnO}_{2}$ nanoparticles as $\mathrm{pH}-/ \mathrm{H}_{2} \mathrm{O}_{2}$ responsive dissociable nanocarriers to modulate tumor hypoxia for effective combination therapy, Adv. Mater., 2016, 28, 7129-7136.

169 W. Zhu, Z. Dong, T. Fu, J. Liu, Q. Chen, Y. Li, R. Zhu, L. Xu and Z. Liu, Modulation of hypoxia in solid tumor microenvironment with $\mathrm{MnO}_{2}$ nanoparticles to enhance photodynamic therapy, Adv. Funct. Mater., 2016, 26, 5490-5498.

170 S. Chen, Q. Jia, X. Zheng, Y. Wen, W. Liu, H. Zhang, J. Ge and P. Wang, PEGylated carbon $\operatorname{dot} / \mathrm{MnO}_{2}$ nanohybrid: a 
new $\mathrm{pH} / \mathrm{H}_{2} \mathrm{O}_{2}$-driven, turn-on cancer nanotheranostics, Sci. China Mater., 2018, 61, 1325-1338.

171 R. Liu, L. Zhang, J. Zhao, Z. Luo, Y. Huang and S. Zhao, Aptamer and IR820 dual-functionalized carbon dots for targeted cancer therapy against hypoxic tumors based on an $808 \mathrm{~nm}$ laser-triggered three-pathway strategy, $A d v$. Ther., 2018, 1, 1800041.

172 D. W. Zheng, B. Li, C. X. Li, J. X. Fan, Q. Lei, C. Li, Z. Xu and $\mathrm{X}$. Z. Zhang, Carbon-dot-decorated carbon nitride nanoparticles for enhanced photodynamic therapy against hypoxic tumor via water splitting, ACS Nano, 2016, 10, 8715-8722.

173 C. Sabu, C. Rejo, S. Kotta and K. Pramod, Bioinspired and biomimetic systems for advanced drug and gene delivery, J. Controlled Release, 2018, 287, 142-155.

174 L. Gao, J. Zhuang, L. Nie, J. Zhang, Y. Zhang, N. Gu, T. Wang, J. Feng, D. Yang, S. Perrett and X. Yan, Intrinsic peroxidase-like activity of ferromagnetic nanoparticles, Nat. Nanotechnol., 2007, 2, 577-583.
175 Y. Huang, J. Ren and X. Qu, Nanozymes: classification, catalytic mechanisms, activity regulation, and applications, Chem. Rev., 2019, 119, 4357-4412.

176 M. Liang and X. Yan, Nanozymes: from new concepts, mechanisms, and standards to applications, Acc. Chem. Res., 2019, 52, 2190-2200.

177 J. Wu, X. Wang, Q. Wang, Z. Lou, S. Li, Y. Zhu, L. Qin and H. Wei, Nanomaterials with enzyme-like characteristics (nanozymes): next-generation artificial enzymes(II), Chem. Soc. Rev., 2019, 48, 1004-1076.

178 F. Liu, L. Lin, Y. Zhang, Y. Wang, S. Sheng, C. Xu, H. Tian and $\mathrm{X}$. Chen, A tumor-microenvironment-activated nanozyme-mediated theranostic nanoreactor for imagingguided combined tumor therapy, Adv. Mater., 2019, 31, 1902885.

179 H. Sun, Y. Zhou, J. Ren and X. Qu, Carbon nanozymes: enzymatic properties, catalytic mechanism, and applications, Angew. Chem., Int. Ed., 2018, 57, 9224-9237. 